\title{
Self-assembled organic and polymer photonic crystals for laser applications
}

\begin{abstract}
Seiichi Furumi ${ }^{1,2,3}$
This article describes an overview of recent developments in fabrication and uses of self-assembled photonic crystals (PCs) of organic and polymer materials, such as chiral liquid crystals (CLCs) and colloidal crystals (CCs), for laser applications. Both CLCs and CCs have intrinsic capabilities to spontaneously assemble 1D-PC and 3D-PC structures, respectively. When a periodic length in the PC structures of CLCs and CCs corresponds to several hundred nanometers of the light wavelength, the photonic band-gaps (PBGs) can be visualized as Bragg reflection colors. When combining fluorescence dyes in the CLCs and CCs, the stimulated laser action at PBG band edge(s) or within the PBG wavelength can be generated by optical excitation. Moreover, the optically excited laser action is controllable by external stimuli due to the self-organization of CLCs and CCs. This review highlights not only the research backgrounds of CLC and CC structures as PCs, but also the experimental results of their versatile soft and tunable laser applications. We believe that a wide variety of CLC and CC structures will have leading roles in the next generation of optoelectronic devices of organic and polymer materials.
\end{abstract}

Polymer Journal (2013) 45, 579-593; doi:10.1038/pj.2012.181; published online 14 November 2012

Keywords: colloidal crystals; chiral liquid crystals; laser; microparticles; photonic band-gap; photonic crystals; self-assembly

\section{INTRODUCTION}

Photonic crystals (PCs) have received a great deal of attention as promising platforms for next generation optoelectronics, because of their intriguing prospects for the on-demand manipulation of not only photons, but also photon-matter interactions within a small space. ${ }^{1}$ In general, PCs consist of periodically modulated structures of different dielectric materials with the periodic length nearly equal to the light wavelength. When a light wave propagates into a PC structure, we can observe the forbidden regions for photons in the dispersion spectrum. Such specific regions are known as 'photonic band-gaps (PBGs)'. The way in which the PCs interact with light waves is analogous to the interaction of atomic crystals with electrons. Interest in the PCs is increasing in both fundamental and practical photonics researches as the concepts were independently reported by Yablonovitch $^{2}$ and $\mathrm{John}^{3}$ in 1987. Yablonovitch established the concept of PBG from a theoretical viewpoint. He noted that spontaneous light emission can be controlled by an energy region in which light cannot propagate in any direction, and used the technical term of PBG. ${ }^{2}$ At the same time, John pointed out the strong localization of photons with certain energies around defects embedded in a dielectric medium by numerical calculation with Maxwell's equations. ${ }^{3}$ Importantly, before the PC concept, Ohtaka has already reported the archetypal term of 'band' for photons. ${ }^{4}$
According to the periodic dimensionality, the PCs can be categorized into three types: one dimensional- (1D-), two dimensional(2D-) and three dimensional- (3D-) PCs (Figure 1). ${ }^{5}$ As evident from this illustration, 1D-PCs-periodically multilayered structures of different dielectric materials - are the simplest in all PC structures (Figure 1a). Nowadays, 1D-PCs are widely used as traditional optical components such as dichroic mirrors, notch filters and antireflection layers. Such 1D-PCs can be obtained by the evaporation process; in this case, two kinds of different dielectric materials are alternately stacked in a repeated manner. Therefore, this fabrication process is very precise and time-consuming to prepare the desired structures and optical properties of 1D-PCs.

2D-PCs consist of columns arranged in an in-plane crystal lattice structure (Figure 1b), and hence, in these 2D-PCs, a light wave propagating in any direction in the plane can be reflected. At present, there have been many reports on not only the fabrication of 2D-PCs by top-down techniques, such as lithography and holography for semiconductor electronic devices, but also the evaluation of unique optical properties by the PBG effect of 2D-PCs. ${ }^{6-8}$ Studies on $2 \mathrm{D}$-PCs with PBGs in near-infrared or visible wavelength regions have been hampered by the difficulty in preparing precisely ordered periodic structures of 2D-PCs with the desired aspect ratios of columns. Recently, several reports have successfully proven that the 2D-PCs

\footnotetext{
${ }^{1}$ Applied Photonic Materials Group, National Institute for Materials Science (NIMS), Ibaraki, Japan; ${ }^{2}$ Nanosystems and Emergent Functions, Precursory Research for Embryonic Science and Technology (PRESTO), Japan Science and Technology Agency (JST), Saitama, Japan and ${ }^{3}$ Department of Materials Science and Engineering, Graduate School of Pure and Applied Sciences, University of Tsukuba, Ibaraki, Japan Correspondence: Dr S Furumi, Applied Photonic Materials Group, National Institute for Materials Science (NIMS), 1-2-1 Sengen, Tsukuba, Ibaraki 305-0047, Japan. E-mail: FURUMI.Seiichi@nims.go.jp

Received 4 July 2012; revised 2 September 2012; accepted 11 September 2012; published online 14 November 2012
} 

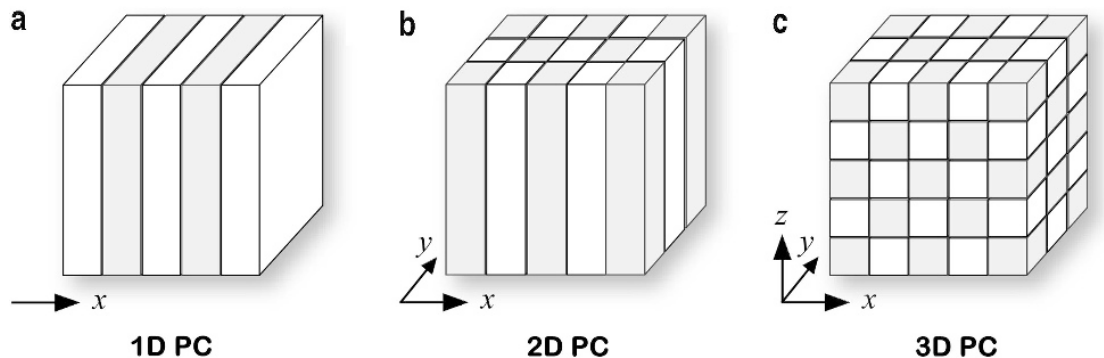

Figure 1 Schematic illustrations of 1D- (a), 2D- (b), and 3D- (c) photonic crystals (PCs).

with near-infrared and visible PBG wavelengths can be prepared by utilizing glass capillary plates ${ }^{9,10}$ and anodic porous alumina. ${ }^{11}$

3D-PCs have spatially lattice structures of building blocks with a scale of light wavelengths, and the most complicated structures in all PCs (Figure 1c). 3D-PCs have an outstanding potential to fully prevent light propagation within a specific $P B G$ wavelength range and in any direction of PCs by the complete PBG (or full PBG). Many reports have been made on a wide variety of methodologies to fabricate 3D-PCs by top-down techniques of lithographic and selective etching, ${ }^{12,13}$ multiphotons polymerization ${ }^{14,15}$ and holographic method. ${ }^{16}$

On the other hand, chiral liquid crystals (CLCs) and colloidal crystals (CCs) have peculiar capabilities to spontaneously assemble 1D-PCs of chiral molecules and 3D-PCs of colloidal microparticles by a bottom-up process, respectively (Figure 2 ). In other words, we do not require any specific facilities for the fabrication of PC structures. The details of CLCs and CCs are described in the following sections. This review deals with not only the research backgrounds of CLC and CC structures as PCs, but also the experimental results of their versatile soft and tunable laser applications.

\section{CLCs AS PCs}

Current trend in the PC research realm is devoted to development of 2D-PC and 3D-PC structures, because they have possibilities to create the complete PGBs. Although theoretical calculation indicates that it is not possible to produce the complete PBGs by $1 \mathrm{D}-\mathrm{PCs}$, the $1 \mathrm{D}-\mathrm{PC}$ structures can be used to generate strong localization of photons within the PGB or at PBG edge(s). Considering the peculiar features, such 1D-PC structures can be applied to intriguing photonic devices as low-threshold lasers, optical amplifiers, and so on. ${ }^{1}$

In this context, CLCs, which are typically supramolecular helical assemblages of nematic liquid crystal (LC) layers with a periodic helical pitch, have garnered considerable attention from the intriguing research field of PCs. ${ }^{17}$ In general, such CLC mesophases appear from intrinsically chiral (optically active) compounds or mixtures where at least one of the components has an enantiomeric chiral center. ${ }^{18}$ In the CLC mesophase, the orientational order of LC molecules is typically similar to that of nematic LC layers. However, the local director of each nematic LC layer spatially and periodically rotates clockwise or counterclockwise with respect to the helical axis, thereby resulting in the formation of right-handed or left-handed supramolecular helical structures of CLCs, respectively.

When the CLC materials are sandwiched between two substrates with homogeneous anchoring surfaces, the CLC supramolecular helical axis is spontaneously aligned to the orthogonal direction of the substrate surface. In this alignment, the CLC cells show a unique optical texture under crossed-nicols, which is so-called Grandjean or planar texture. ${ }^{18}$ Owing to the supramolecular helical structures, the

\section{a Chiral liquid crystals (CLCs)}

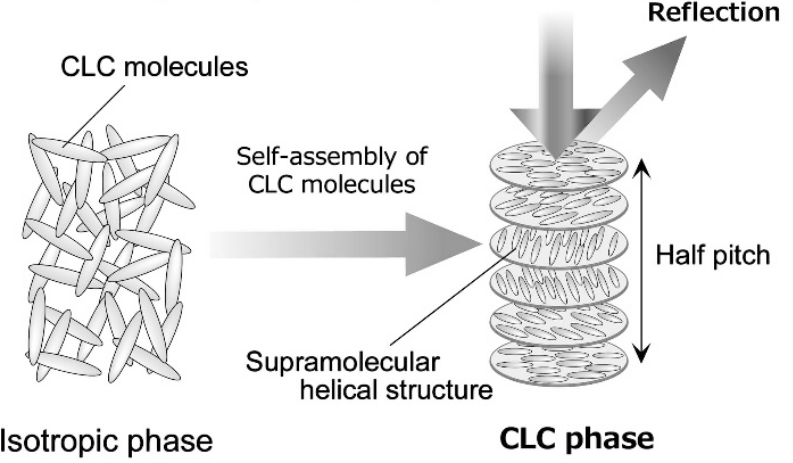

\section{b Colloidal crystals (CCs)}

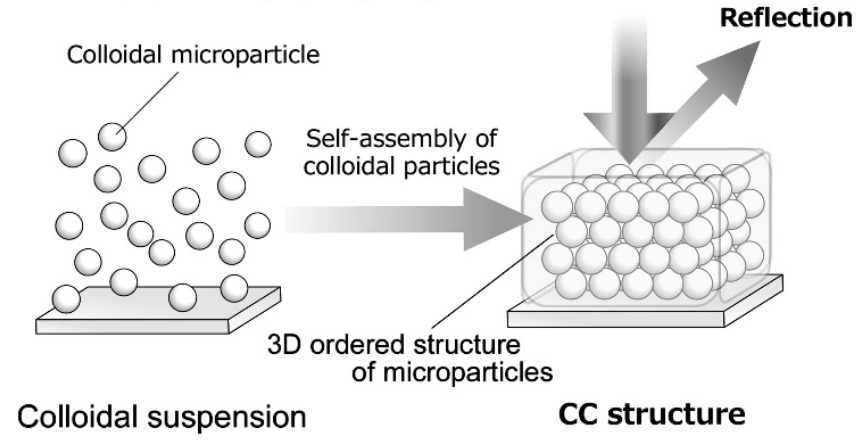

Figure 2 Schematic illustrations of self-assembled organic and polymer PCs of chiral liquid crystals (CLCs) (a) and colloidal crystals (CCs) (b). Both CLCs and CCs have intrinsic capabilities to spontaneously assemble 1D- and 3D-PC structures by bottom-up process, respectively.

planar-aligned CLC cell exhibits negative birefringence with the extraordinary refractive index $\left(n_{\mathrm{e}}\right)$ along the CLC helical axis. The light reflection is the most unique and important optical property of CLCs. ${ }^{18}$ The maximum wavelength of light reflection $\left(\lambda_{\max }\right)$ is numerically expressed as the following equation:

$$
\lambda_{\max }=n_{\mathrm{av}} p
$$

where $n_{\mathrm{av}}$ represents the average optical refractive index and $p$ is the helical pitch length of CLC material. The reflection bandwidth $(\Delta \lambda)$ can be determined by the following expression:

$$
\Delta \lambda=\Delta n p=\left(n_{\mathrm{e}}-n_{\mathrm{o}}\right) p
$$

where $\Delta n$ is the optical anisotropy and $n_{\mathrm{o}}$ is the ordinary refractive index of CLC.

When a planar-aligned CLC cell is observed at oblique incidence, the wavelength of reflection light is depended on the incident angle 
$(\theta)$. The reflection wavelength is changed according to the following trigonometric function:

$$
\lambda_{\max }(\theta)=n_{\mathrm{av}} p \cos \theta
$$

where $\lambda_{\max }(\theta)$ is the reflection wavelength at $\theta$ from the surface normal of planar-aligned CLC cell. When observing at oblique incidence, the reflection wavelength shifts to shorter wavelengths.

Another outstanding characteristic of the CLCs is that the light reflection shows chiroptical (chiral optical) property. When linearly polarized light propagates into a planar-aligned CLC cell along the helical axis, the CLC molecules can reflect circularly polarized light (CPL) with same CLC supramolecular helical handedness at a wavelength of $\lambda_{\max }$ with a bandwidth of $\Delta \lambda$. On the other hand, CPL with the opposite CLC helical handedness transmits through the planar-aligned cell without light reflection. In this way, the lightreflecting behavior of CLCs depends on the CPL state, which is socalled 'selective light reflection'. When the selective light reflection takes place in a visible wavelength range, the helical pitch of CLCs is of the order of several hundred nanometers, and the optical rotation power is $10^{3}-10^{5}$ degree $\mathrm{mm}^{-1}$.

Figure 3 shows the experimental results of a polymerized CLC film with left-handed supramolecular helical sense. ${ }^{19}$ Polarized microscopic observation confirms that the CLC film shows Grandjean texture, in which the helical axis is uniformly perpendicular to the substrate surface (inset in Figure 3a). The CPL transmission spectra are probed by using right-handed CPL (R-CPL) and left-handed CPL (L-CPL). As shown in Figure 3a, the spectral shape is drastically altered by changing the CPL direction of probing light between $\mathrm{R}-\mathrm{CPL}$ and L-CPL. When the R-CPL is adopted to measure the CPL transmission spectrum, this polymerized CLC film exhibits transparency in a wavelength range from 500 to $900 \mathrm{~nm}$ (gray curve in Figure 3a). When the probing light is switched from R-CPL to L-CPL, a characteristic band emerges in a wavelength range from 570 to $600 \mathrm{~nm}$ due to the selective light reflection by the CLC film (black curve in Figure 3a). This reflection band appears by the lefthanded supramolecular helical sense of this polymer CLC. When the transmission spectra are monitored at angles of oblique incidence, this reflection band shifts to shorter wavelengths in line with equation (3).

Scanning electron microscopy observation of this polymerized CLC film allows us to visualize the spatial structure of CLC molecules at the microscopic level. Figure $3 \mathrm{~b}$ shows a cross-sectional scanning electron microscopy image of this CLC film. As is evident from this scanning electron microscopy image, the periodically multilayered structure is aligned perpendicularly to the substrate surface. This periodicity corresponds to a half length of helical pitch of this planaraligned polymer CLC film. The empirical pitch length is estimated to be $\sim 360 \mathrm{~nm}$, and the average optical refractive index of this CLC is $\sim 1.61$. By importing the $p$ and $n_{\mathrm{av}}$ values in equation (1), the calculated reflection wavelength of $\sim 580 \mathrm{~nm}$ is found to be in substantially good agreement with the experimental reflection spectrum.

Such CLC materials with unique reflection properties have been long-standing subject with considerable attention for their technological applications such as display devices, full-color recording media, polarizers and reflectors. ${ }^{20-25}$ To date, mirrorless laser action from the dye-doped CLCs has been frequently investigated for use in the molecular photonic devices. ${ }^{17}$ The following sections outline the typical experimental results of laser action behaviors of CLCs by optical excitation.
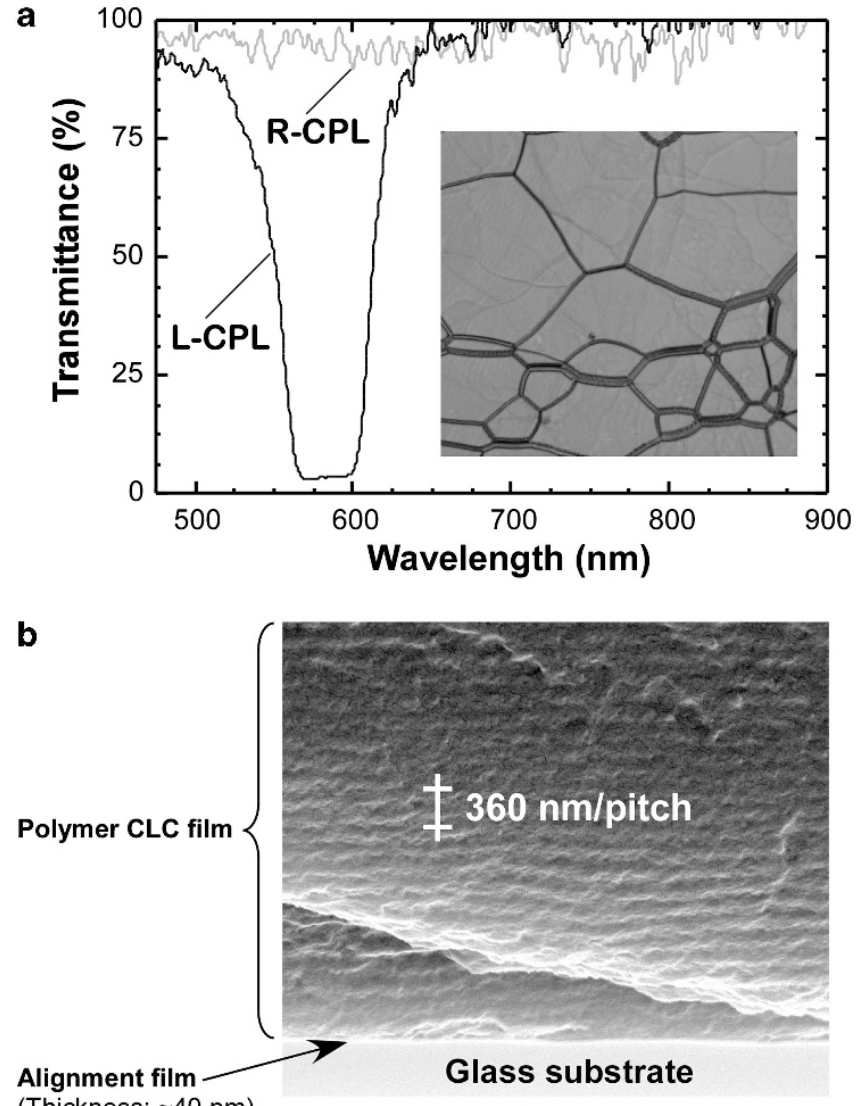

(Thickness: $\sim 40 \mathrm{~nm}$ )

Figure 3 (a) Circularly polarized light (CPL) transmission spectra of a planar-aligned polymer chiral liquid crystals (CLC) film with left-handed supramolecular helical sense. Black and gray curves denote the transmission spectra probed by left-handed $\mathrm{CPL}(\mathrm{L}-\mathrm{CPL})$ and right-handed $\mathrm{CPL}$ (R-CPL), respectively. The inset shows Grandjean optical texture of the planar-aligned CLC cell under crossed-Nicols. (b) Scanning electron microscopy image observed from cross-sectional direction of this planaraligned polymer CLC cell. Reproduced with permission from Furumi. ${ }^{19}$

\section{LASER ACTION FROM DYE-DOPED CLCs}

Recently, numerous advances of CLCs in terms of 1D-PCs have established a new perspective in the photonic research field. In particular, mirrorless laser action can be generated by optical excitation of the CLCs doped with a fluorescent dye. ${ }^{17,19,26-28}$ Generally, the laser action takes place at the edge(s) of the CLC reflection band, corresponding to the PBG, due to the internal laserfeedback effect. Four decades ago, the original concept of CLC lasers has been reported by Goldberg and Schnur in a US patent. ${ }^{29}$ As far as we know, Kopp et al. ${ }^{30}$ demonstrated for the first time the laser emission from a CLC cell doped with a fluorescent pyrromethene derivative by optical excitation. ${ }^{30}$

Following the first demonstration of optically excited laser action from CLCs, the stimulated laser emission from CLC materials has become one of the leading research topics for fabrication of new organic and polymer photonic devices. Hitherto, there have been numerous reports on the laser action from various CLC materials, such as low molecular weight $\mathrm{CLCs}^{30-33}$ and ferroelectric LCs, ${ }^{34}$ polymerized CLCs, ${ }^{35-38}$ and lyotropic CLCs. ${ }^{39}$ Among a variety of CLC mesophases, cholesteric blue-phase LCs are made of unique 3D cubic structures of chiral LC molecules with lattice periods of several hundred nanometers. ${ }^{40}$ Such blue-phase typically appears in a narrow 
temperature range by precise cooling process from the isotropic phase. In previous studies, optically excited laser action are successfully demonstrated by using low molecular weight bluephase LCs, ${ }^{41}$ as well as polymer stabilized blue-phase LCs. ${ }^{42}$

The typical experimental results of optically excited laser action from the CLCs doped with a fluorescent dye are described below (Figure 4) ${ }^{43}$ In order to prepare the CLC hosts with PBGs in a visible wavelength range, we use two kinds of compounds of RDP-60774 as an achiral nematic LC and R-1011 as a chiral dopant (Figure 4a). This LC of RDP-60774 exhibits nematic LC phase up to $43^{\circ} \mathrm{C}$, and comprises a binary mixture of phenycylcohexane derivatives with a cyano group at their terminal positions. Therefore, the dielectric anisotropy $(\Delta \varepsilon)$ is a relatively high value of +11.3 , as compared with those of other nematic LCs. A chiral dopant of R-1011 has a chiral center tethering two mesogen moieties, and its helical twisting power $(\beta)$ is $42.1(\mu \mathrm{mwt} \%)^{-1}$. When $6.0 \mathrm{wt} \%$ of $\mathrm{R}-1011$ is mixed in RDP60774 , corresponding to $\sim 2.0 \mathrm{~mol} \%$, the CLC host shows a reflection band around $600 \mathrm{~nm}$. After dissolving R-1011 in RDP$60774,0.4 \mathrm{wt} \%$ of a fluorescent dye, 4-(dicyanomethylene)-2-methyl6-(4-dimethylaminostryl)-4H-pyran (DCM; inset in Figure $4 \mathrm{~b}$ ), is added into the CLC host. This DCM dye shows a broad fluorescence spectrum centered at $590 \mathrm{~nm}$ by optical excitation with $532 \mathrm{~nm}$ light from an Xe lamp (Figure 4b). This DCM-doped CLC mixture is sandwiched between indium tin oxide glass substrates covered with uniaxially rubbed poly(vinyl alcohol) films to induce planar CLC alignment.

Reflection spectral measurement reveals that a selective reflection band of this CLC host appears in a wavelength range from 580 to $630 \mathrm{~nm}$ (dashed curve in Figure 4c). This reflection band completely overlaps with a broad fluorescence band of the DCM dye (Figure 4b). The laser emission characteristics of this DCM-doped CLC cell are evaluated by using the second harmonic generation light at $532 \mathrm{~nm}$ emitted from a $Q$-switched Nd:yttrium aluminum garnet laser beam with a pulse width of $\sim 6 \mathrm{~ns}$. When this DCM-doped CLC cell is excited with a relatively low energy up to $\sim 0.9 \mu \mathrm{J}$ per pulse, the emission intensity monotonously increases with the excitation energy. At an excitation energy of $0.9 \mu \mathrm{J}$ per pulse, we can observe the following anomalous alteration in a broad fluorescence spectrum of DCM dye: the emission is inhibited within a wavelength range of CLC selective reflection band. This fluorescence spectral alteration stems from the strong localization of emitting photons from DCM within the selective reflection band of CLC host. In other words, the reflection band of CLC host works as the PBG. When the excitation energy exceeds a threshold excitation energy of $0.9 \mu \mathrm{J}$ per pulse, the emission intensity is increasingly intensified, accompanied by the abrupt spectral narrowing from 80 to $0.8 \mathrm{~nm}$ (solid curve in Figure $4 \mathrm{c}$ ). This laser emission peak emerges at $\sim 630 \mathrm{~nm}$ corresponding to the longer-wavelength edge of this CLC selective reflection band.

Taking account of the fact that the laser emission peak clearly appears at the CLC reflection band edge, we prepare DCM-doped CLC cells with $6.0 \mathrm{wt} \%, 5.9 \mathrm{wt} \%$ and $5.4 \mathrm{wt} \%$ of R-1011 in RDP60774. Figures $4 \mathrm{c}-\mathrm{e}$ show the reflection and laser emission spectra. The selective reflection band shifts to shorter wavelengths as increasing the concentration of R-1011 (dashed curves in Figures 4c-e). This behavior obeys the following equation. ${ }^{44}$

$$
\lambda_{\max }=n_{\mathrm{av}}(\beta C)^{-1}
$$

where $\beta$ stands for the helical twisting power and $C$ is the concentration of R-1011 in RDP-60774. This equation can be applied when the concentration of chiral dopant is low. The laser emission

\section{a RDP-60774}

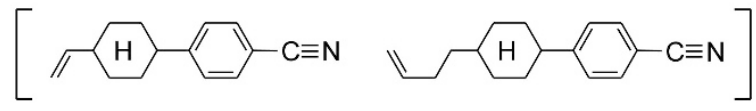

\section{R-1011}
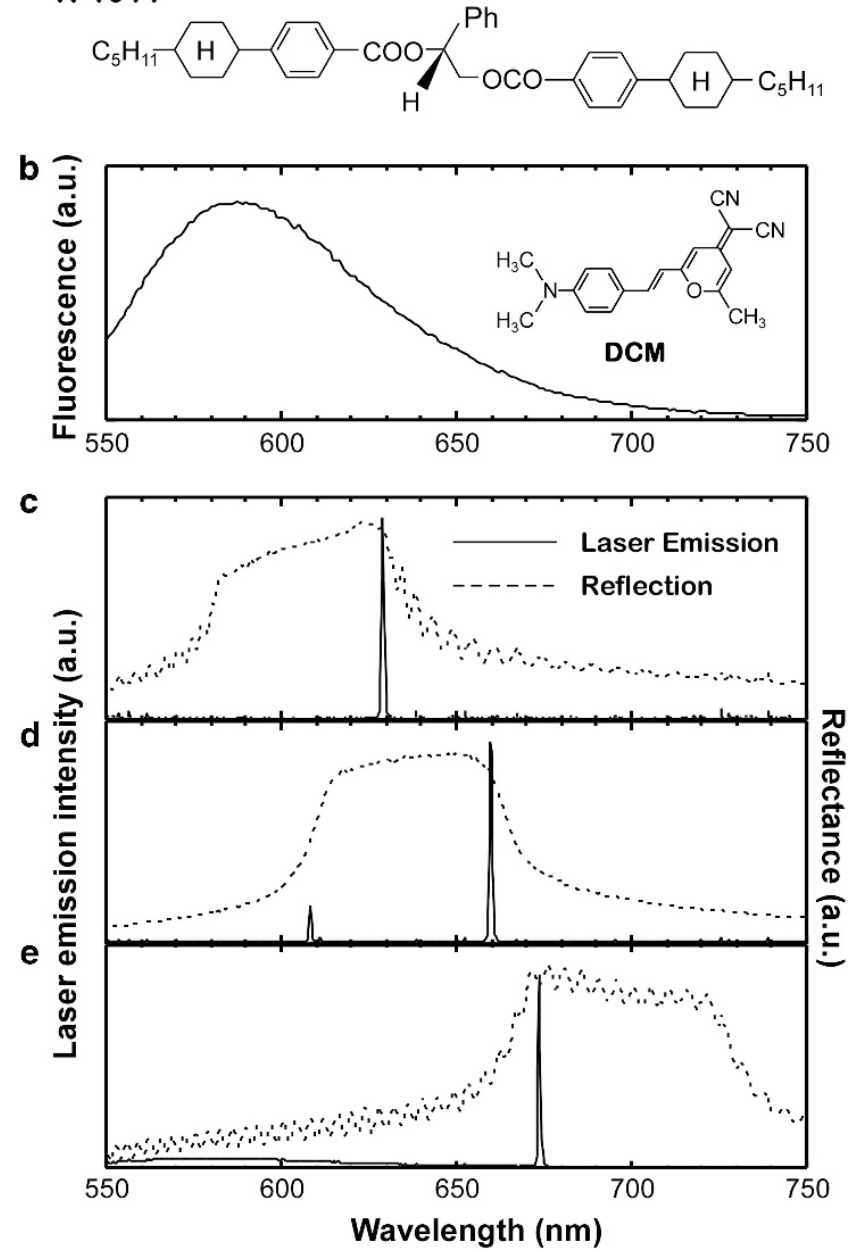

Figure 4 (a) Chemical structures of achiral nematic LC (RDP-60774) and chiral agent (R-1011). (b) Fluorescence spectrum of a fluorescent dye (DCM) in chiral liquid crystals (CLC) by optical excitation with $532 \mathrm{~nm}$ light from a Xe lamp. The inset shows the chemical structure of DCM. (c-e) Optically excited laser emission (solid curves) and reflection (dashed curves) spectra of DCM-doped CLC cells, filled with $6.0 \mathrm{wt} \%$ (c), 5.9 wt\% (d) and 5.4 wt\% (e) of R-1011 in RDP-60774. The laser emission spectra were measured by optical excitation with $532 \mathrm{~nm}$ light from Nd:yttrium aluminum garnet laser beam with a pulse energy of $\sim 1.2 \mu \mathrm{J}$ per pulse. Reproduced with permission from Furumi ${ }^{19}$ and Furumi et al. ${ }^{43}$

peak is significantly dependent on the wavelength position of the selective reflection band of CLC host (solid curves in Figures $4 \mathrm{c}-\mathrm{e}$ ). A single laser emission peak observed from a DCM-doped CLC cell with $6.0 \mathrm{wt} \%$ of R-1011 appears at the longer-wavelength edge of CLC reflection band $(630 \mathrm{~nm}$; Figure $4 \mathrm{c})$, whereas a CLC cell with $5.4 \mathrm{wt} \%$ of R-1011 shows a single laser emission peak at the shorterwavelength edge ( $675 \mathrm{~nm}$; Figure $4 \mathrm{e})$. Very interestingly, when the CLC cell is prepared by mixing $5.9 \mathrm{wt} \%$ of R-1011 in RDP-60774, two laser emission peaks can be observed at both the shorter- and longerwavelength edges of the CLC reflection band (608 and $658 \mathrm{~nm}$; Figure $4 \mathrm{~d}$ ). The two laser emission peaks probably arise because the selective reflection band is entirely covered by the broad fluorescence 
spectrum of DCM above the fluorescence maximum wavelength (Figures $4 \mathrm{~b}$ and $\mathrm{d}$ ).

\section{CIRCULAR POLARIZATION OF LASER ACTION FROM CLCS}

Polarization control of emission properties of organic and polymeric materials has attracted considerable interest from technological viewpoints because of its advantages in high-density photonic devices. One of interesting research subjects is the generation of CPL emission-emitting light with different intensities for R-CPL and L-CPL components - by the intrinsic structural asymmetry of chiral molecules. This research progress lags behind that of linearly polarized emission. This is because of the relative difficulties in preparing a helical molecular arrangement by conventional techniques such as mechanical stretching and rubbing for uniaxial molecular alignment. It is common knowledge that introduction of enantiomerically pure chiral compounds or groups in a molecular system leads to the chiroptical properties at the ground state such as optical rotatory dispersion and circular dichroism. In contrast, the chiroptical properties of CPL emission at the excited state cannot be generated in straightforward ways. Intriguing attempts have been made to increase the degree of CPL emission by synthesizing $\pi$-conjugated polymers with chiral side chains, ${ }^{45-49}$ chiral metal complexes, ${ }^{50-53}$ and helicene derivatives with steric hindrance ${ }^{54,55}$

On the other hand, CLC materials are readily available to efficiently generate CPL emission. When a fluorescent dye is dissolved in the CLC material, the selective reflection band superimposes on the broad fluorescence band emitted from the guest dye. So far, there have been lots of reports on the CPL broad emission with opposite handedness to the supramolecular helical sense of CLC host by optical excitation with non-polarized light. ${ }^{56-59}$ However, the CPL degrees by these techniques are not sufficient for practical applications in dissymmetric light sources.

As mentioned in the preceding section, the CLCs show an outstanding chiroptical property of selective light reflection. From the standpoint of the PC research field, the photon dispersion diagrams of CLCs are quite different from those in the conventional 1D-PCs such as periodically multilayered structures. When linearly polarized or non-polarized light propagates into a planar-aligned CLC cell, the light wave can be divided into two CPL components of $\mathrm{R}-\mathrm{CPL}$ and L-CPL. Although the CPL component with same CLC helical handedness is reflected by the selective reflection band, the other CPL component with opposite handedness is transmitted. Therefore, it can be predicted that the optically excited laser action would show circular polarization features depending on the supramolecular helical handedness of CLC host.

In this section, the CPL characteristic of laser action from a dyedoped CLC cell is evaluated by the combination of a quarter-wave plate and a linear polarizer. Figure 5 shows the L-CPL and R-CPL laser emission spectra from a DCM-doped CLC cell fabricated with $5.9 \mathrm{wt} \%$ of R-1011 in RDP-60774. As is evident from the spectra, the laser emission peaks observed from this CLC cell have predominant $\mathrm{R}-\mathrm{CPL}$ component rather than L-CPL. This is because this CLC host doped with R-1011 has a right-handed supramolecular helical sense. Generally, the circular polarization degree of emission $\left(g_{\mathrm{em}}\right)$ is given by the following equation. ${ }^{60}$

$$
g_{\mathrm{em}}=2\left(I_{\mathrm{L}}-I_{\mathrm{R}}\right) /\left(I_{\mathrm{L}}+I_{\mathrm{R}}\right)
$$

where $I_{\mathrm{L}}$ and $I_{\mathrm{R}}$ stand for the emission intensities of L-CPL and $\mathrm{R}-\mathrm{CPL}$, respectively. In the case of purely single-handed CPL emission, the $g_{\mathrm{em}}$ value shows +2 or -2 for the L-CPL or R-CPL emission, respectively. From CPL laser emission spectra (Figure 5),

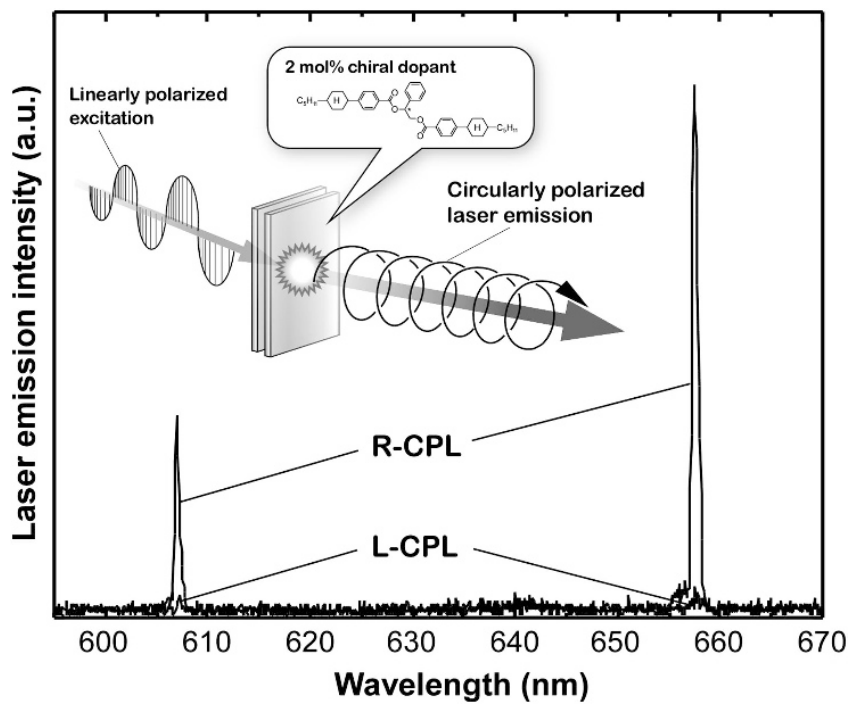

Figure 5 Circularly polarized laser (CPL) emission spectra from a DCMdoped chiral liquid crystal (CLC) cell, filled with $5.9 \mathrm{wt} \%$ of R-1011 in RDP-60774. The chemical structures of RDP-60774, R-1011 and DCM are shown in Figure 4. R-CPL and L-CPL denote the right-handed and left-handed CPL of emission spectra, respectively. The inset represents the illustration of circularly polarized laser emission from the dye-doped CLC cell by optical excitation with a linearly polarized light. Reproduced with permission from Furumi. ${ }^{19}$

the $g_{\text {em }}$ values of both laser emission peaks at 608 and $658 \mathrm{~nm}$ are calculated to be -1.85 . This $g_{\mathrm{em}}$ value is higher than those observed for other CLC systems. ${ }^{56-59}$

This experimental result motivates the investigation of CPL laser emission from a CLC cell doped with an enantiomeric (mirror-image) chiral dopant of R-1011, that is, S-1011. By mixing S-1011 and RDP60774, the CLC host exhibits left-handed supramolecular helical sense. As a result, L-CPL laser emission is observed for a DCMdoped CLC cell with S-1011. This result indicates that the CPL direction of laser emission by CLC materials can be controlled by appropriate choice of an enantiomeric chiral dopant dissolved in the achiral nematic LC, in spite of a very small amount of chiral dopant ( $2.0 \mathrm{~mol} \%)^{61,62}$

In this way, the laser emission from the dye-doped CLC cell exhibits the chiroptical property with an almost single CPL handedness, even though the excitation beam is linearly polarized (inset in Figure 5). Most interestingly, the fluorescent DCM dye does not have molecular chirality by itself, namely the achiral molecule. It should be stressed that the CLC host enables the generation of CPL laser emission from achiral fluorescent dye by the chiral PBG of CLC host.

\section{ELECTRICAL CONTROL OF LASER ACTION FROM CLCs}

The most important property of functional LC materials is capabilities not only to induce substantial enhancement of their anisotropic physical properties by the molecular assemblages and alignment, but also to control the anisotropic properties by external stimuli. ${ }^{63,64}$ In the case of CLCs, selective light reflection properties of low molecular weight CLCs can be controlled by external stimuli such as temperature, pressure, electric field and so forth. ${ }^{18}$ Previously, we succeeded in the electrical control of supramolecular helical structure and laser action from electroactive CLCs by applying voltages. ${ }^{43,65}$ This section introduces some of experimental results of the electrically controlled lasing action. 
Dynamic control of laser emission is realized by applying external voltages with an alternating current to the aforementioned DCMdoped CLC cell. The achiral nematic RDP-60774 to prepare the CLC host has a positive and relatively high $\Delta \varepsilon$ value of +11.3 (Figure $4 \mathrm{a}$ ). Figure 6 shows the changes in laser emission intensity from the optically excited CLC cell as a function of the applied voltage. ${ }^{43}$ Although the laser emission from the DCM-doped CLC cell can be constantly observed up to $18 \mathrm{~V}$, applying a voltage of $20 \mathrm{~V}$ immediately depletes the intensity of laser emission. At voltages higher than $20 \mathrm{~V}$, we confirm the presence of the selective reflection band of CLC host. However, the laser emission is not generated at all even when the excitation energies are set at $5.0 \mu \mathrm{J}$ per pulse or more. This response can be attributed to the change in reflection band edges of CLC host. Both the shorter- and longer-wavelength edges of the reflection band disappear by applying a voltage of $20 \mathrm{~V}$ (inset in Figure 6).

Such lasing behavior can be unraveled by polarized optical microscopic observation upon increasing the external voltages. Initial Grandjean texture of CLC cell is converted into focal conic texture in which the orientation of CLC helical axis is random. ${ }^{66,67}$ By considering overall results, the planar alignment of CLC host with clear reflection band edges is a prerequisite condition for the generation of optically excited laser action from the dye-doped CLC cell. Upon increasing the applying voltages, the reflection band gradually decreases, and eventually disappears at $70 \mathrm{~V}$ (inset in Figure 6). Polarized optical microscopic observation under crossedNicols reveals that no light is transmitted on applying a voltage of $70 \mathrm{~V}$. This suggests the homeotropic orientation in which the optical axis of LC molecules orients perpendicularly to the substrate surface. In other words, applying such a high voltage unwinds the helical molecular arrangement of the CLC host, resulting in homeotropic alignment of nematic LC through the electrically induced phase change from cholesteric to nematic LC. Moreover, when such high

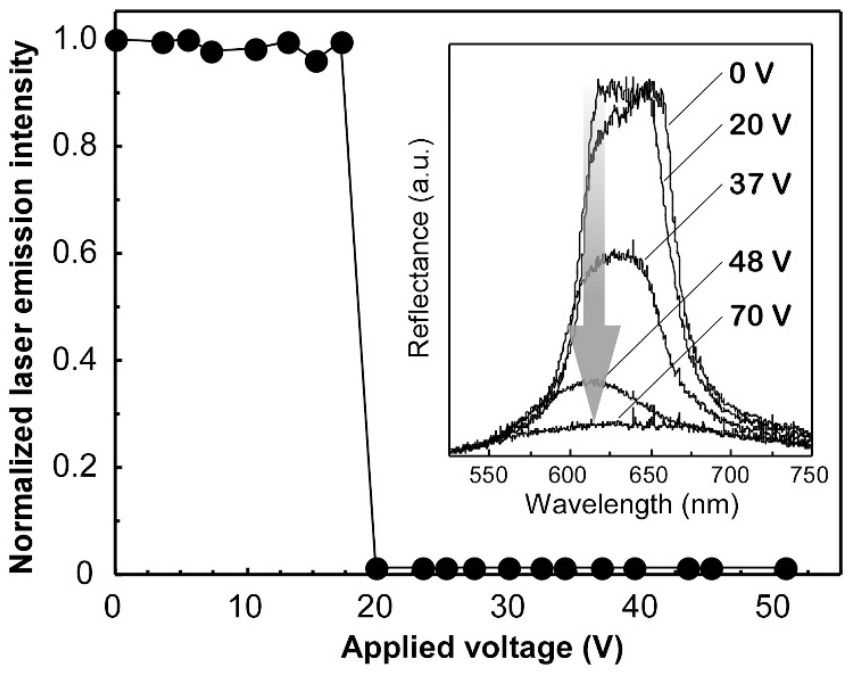

Figure 6 Changes in laser emission intensity from a DCM-doped chiral liquid crystal (CLC) cell, fabricated with 5.9 wt\% of R-1011 in RDP-60774, as a function of applied voltages with an alternating current. The chemical structures of RDP-60774, R-1011 and DCM are shown in Figure 4. The emission intensity was measured upon optical excitation with $532 \mathrm{~nm}$ light with a pulse energy of $\sim 1.9 \mu \mathrm{J}$ per pulse. The inset shows the continuous changes in reflection spectra of the DCM-doped CLC cell upon applying voltages. Reproduced with permission from Furumi ${ }^{19}$ and Furumi et al. ${ }^{43}$ voltage is switched off, the laser emission peak can revive as a consequence of reconstruction of the initial planar CLC alignment. In this way, the laser action can be controlled reversibly by applying a voltage to the electroactive CLC doped with a fluorescent dye.

\section{PHOTOTUNABLE LASER ACTION FROM CLCS}

So far, much attention has converged on the photochemical control of reflection properties of CLCs for technological development of photonic devices such as displays, full-color recording media, and so forth. ${ }^{21,22}$ This situation motivates us to fabricate the photoactive laser devices by photochemical reactions. This section deals with phototunable laser action from a photoreactive CLC cell doped with a fluorescent dye. ${ }^{68}$

In order to prepare a photoreactive CLC, we use three kinds of cholesteryl derivatives: cholesteryl nonanoate, cholesteryl iodide (CI), and cholesteryl oleyl carbonate (Figure 7a). By mixing cholesteryl

a

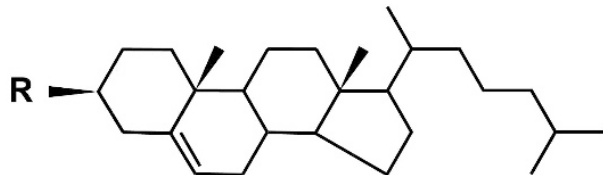

$$
\begin{aligned}
& \mathrm{CN}: \mathrm{R}=\mathrm{C}_{8} \mathrm{H}_{17} \mathrm{COO} \quad \mathrm{Cl}: \mathrm{R}=\mathrm{I} \\
& \text { COC: } \mathrm{R}=\mathrm{C}_{8} \mathrm{H}_{17} \mathrm{CH}=\mathrm{CHC}_{8} \mathrm{H}_{16} \mathrm{OCOO}
\end{aligned}
$$

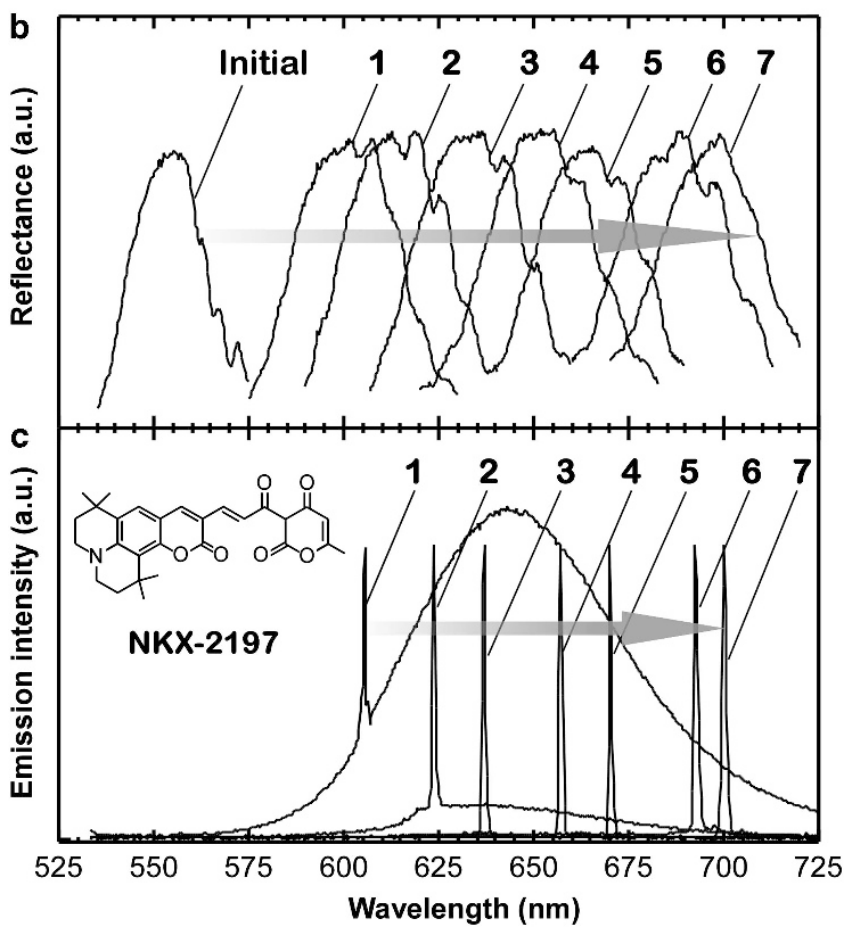

Figure 7 (a) Chemical structures of cholesteryl nonanoate (CN), cholesteryl iodide $(\mathrm{Cl})$, and cholesteryl oleyl carbonate (COC) for preparation of a photoreactive CLC. (b, c) Reflection $\mathbf{b}$ and laser emission spectra $\mathrm{c}$ of dye-doped photoreactive CLC cells with various photonic band-gap (PBG) wavelengths, which were adjusted in advance by irradiation with UV light of $200 \mathrm{~mJ} \mathrm{~cm}^{-2}$ (curve 1), $300 \mathrm{~mJ} \mathrm{~cm}^{-2}$ (curve 2), $400 \mathrm{~mJ} \mathrm{~cm}^{-2}$ (curve 3), $500 \mathrm{~mJ} \mathrm{~cm}^{-2}$ (curve 4), $600 \mathrm{~mJ} \mathrm{~cm}^{-2}$ (curve 5), $700 \mathrm{~m} \mathrm{~cm}^{-2}$ (curve 6) and $800 \mathrm{~mJ} \mathrm{~cm}^{-2}$ (curve 7). The inset shows the chemical structure of a fluorescent benzopyrano quinolizine derivative used in this study. Reproduced with permission from Furumi ${ }^{19}$ and Furumi et al. ${ }^{68}$ 
nonanoate, CI and cholesteryl oleyl carbonate at a weight ratio of 3.0:2.0:2.0, respectively, the photoreactive CLC shows cholesteric LC phase with left-handed supramolecular helical sense up to $56^{\circ} \mathrm{C}$. A planar-aligned CLC cell of the photosensitive CLC shows a reflection band of PBG in a wavelength range of $545-555 \mathrm{~nm}$. Subsequently, the CLC cell is irradiated with ultraviolet (UV) light at $254 \mathrm{~nm}$. The selective reflection band of CLC progressively shifts to longer wavelength in proportion to the exposure energy of UV light due to a photolysis reaction of CI. ${ }^{69}$ Nuclear magnetic resonance spectral measurement confirms that signal intensity of a terminal proton attached to CI monotonously decreases with exposure to UV light. Consumption of $20 \%$ in molar ratio of CI by exposure to UV light of $1.0 \mathrm{~J} \mathrm{~cm}^{-2}$ results in a large shift of the selective reflection band of CLC from 550 to $720 \mathrm{~nm}$. Likewise, a similar shift of selective reflection band is observed for a CLC cell doped with $0.4 \mathrm{wt} \%$ of a fluorescent benzopyrano quinolizine derivative (NKX-2197; inset in Figure 7c). The reflection spectral changes are shown in Figure $7 \mathrm{~b}$.

Successively, we demonstrate the phototuning of the laser emission peak from an NKX-2197-doped CLC cell by exposure to UV light. Figures $7 \mathrm{~b}$ and $\mathrm{c}$ show the changes in reflection and laser emission spectra of the dye-doped CLC cell. A single laser emission peak is clearly determined by the phototuned reflection band position of CLC host. Irradiation of the CLC cell with UV light leads to the precise tuning of a single lasing peak from 610 to $700 \mathrm{~nm} .{ }^{68}$ The laser emission peak always occurs at the longer wavelength edge of CLC reflection band for each cell. It is predictable that the fluorescent NKX-2197 dye is well aligned parallel with the local molecular alignment direction of CLC molecules. In contrast, such lasing action cannot be generated for the dye-doped CLC cells exposed to UV light with the energies of $800 \mathrm{~mJ} \mathrm{~cm}^{-2}$ or more. As the selective reflection band of CLC host scarcely overlaps with the optical gain spectrum of NKX-2197. The phototuned laser action is adequately stable and unchanged as long as it is kept at room temperature for several months. In this way, we successfully demonstrate that the CLC reflection band can be modulated by exposure to UV light, resulting in the on-demand phototuning of laser emission peaks in a widely visible range. Unfortunately, this phototuning process is irreversible. However, the other reports have been successfully made on the photoreversible switching of laser emission peaks by combining photochromic compounds such as azobenzene derivatives in the CLC media. ${ }^{70-72}$ It is greatly advantageous to realize the reversible photocontrol of the laser emission peak for the next-generation photonics.

\section{GLASS-FORMING CLC FOR TUNABLE SOLID-STATE LASER}

Tunable laser of CLC materials is one of the leading research topics from a practical viewpoint. There have been hitherto a wide variety of methodologies to tune the laser emission peak by external stimuli of temperature, mechanical stress, electric field and chiral dopant concentration. ${ }^{19}$ In the traditional distributed feedback lasers by holographic excitation, the subtle control of incident angle of optical excitation beam in a narrow range of $\sim 5^{\circ}$ is definitely required to fine-tune the laser emission peak. ${ }^{73}$ On the other hand, the tunable CLC laser systems are very simple and sophisticated procedures to tune the laser emission peak by control of the selective reflection band of the CLC host.

Recently, more emphasis is placed on the position-dependent tuning of laser emission peaks from continuously-gradated PBG (CG-PBG) structures in planar-aligned CLC cells. ${ }^{74-76}$ In other words, the CG-PBG structures show 1D gradation in the CLC reflection band. To date, the CG-PBG structures of CLCs have been prepared by precisely controlling temperature ${ }^{74}$ and chiral dopant concentration $^{75,76}$ of low molecular weight CLCs. However, the strategies might encounter some serious obstacles as follows. In principle, the low molecular weight CLCs are very vulnerable to subtle temperature fluctuations. When the position-dependent tuning of laser emission is performed by gradating temperature, specific thermal controllers and stages are needed to keep the CG-PBG structures during the lasing experiments. ${ }^{74}$ Moreover, the CG-PBG structures are not stable due to thermodynamic molecular diffusion of the low molecular weight chiral dopants. Therefore, the low molecular weight CLCs are impractical for position-dependent tuning of laser emission peaks. Recently, the CG-PBG structures of photopolymerized CLCs have been prepared for the positiondependent tuning of laser emission. ${ }^{77,78}$ However, the CG-PBG structures require high threshold excitation energies for laser action after photopolymerization. This is because the initial planar CLC alignment or fluorescent dyes might be deteriorated during photopolymerization of CLC monomers. ${ }^{77-79}$

This section presents the preparation of a fluorescent glass-forming CLC (G-CLC) and its application in a new type of tunable CLC solidstate laser. ${ }^{80}$ We succeeded herein in the relatively easy fabrication of robust CG-PBG structures of a fluorescent G-CLC without any covalent bonding by a supercooling process. This CG-PBG structure of G-CLC enables continuously and reversibly tunable laser action by low-threshold optical excitation.

For this purpose, we design and synthesize three kinds of compounds of CD8, 11-BP and DC-OPV to obtain a fluorescent G-CLC. The chemical structures are shown in Figure 8. First, CD8 is used as a G-CLC host. Previously, Tamaoki et al. ${ }^{21,22}$ serendipitously found unique properties of CD8; this CD8 oligomer can be practically applied to rewritable full-color recording devices. A CD8 film shows a selective reflection band of $\mathrm{PBG}$ in a visible wavelength range from 420 to $610 \mathrm{~nm}$, which is dependent on the CLC mesophase temperature between $87^{\circ} \mathrm{C}$ and $115^{\circ} \mathrm{C}$. This $\mathrm{CD} 8$ film annealed at CLC temperatures is rapidly cooled to $0{ }^{\circ} \mathrm{C}$, whereupon the selective light reflection can be preserved even at room temperature for a long time. Such supercooling treatment of CD8 leads to durable formation of a glassy solid-state without any covalent bonding, because of its relatively high glass transition temperature of $\sim 80^{\circ} \mathrm{C}$. This solidified CD8 film has unique abilities for not only the desired tuning of PBG wavelength by annealing temperature, but also the on-demand

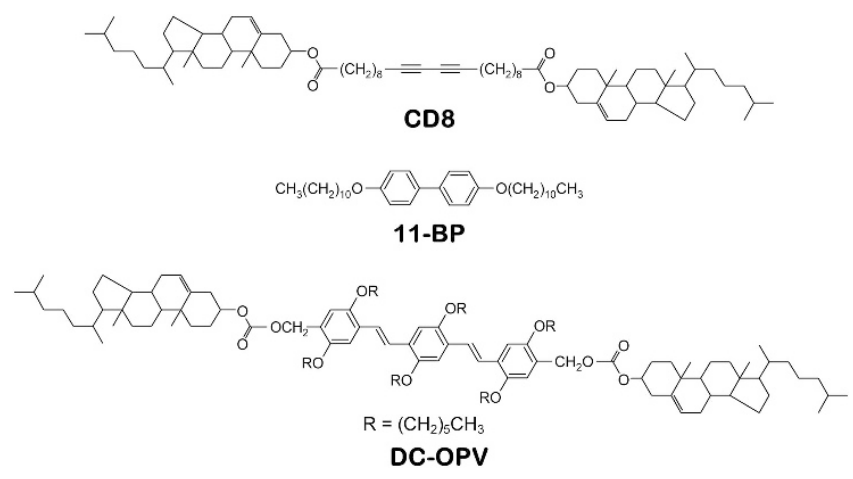

Figure 8 Chemical structures of glass-forming chiral liquid crystal (G-CLC) for fabrication of a new type of tunable solid-state laser. The G-CLC was prepared by mixing ternary compounds of CD8, 11-BP and DC-OPV at a weight ratio of $98.4: 1.6: 1.0$, respectively. Reproduced with permission from Furumi $^{19}$ and Furumi and Tamaoki. ${ }^{80}$ 
preservation of the shifted PBG wavelength by the subsequent supercooling treatment. Secondly, 11-BP is adopted as a subsidiary dopant in order to regulate the PBG wavelength and its tuning range of CD8. Thirdly, we synthesize an oligo( $p$-phenylene vinylene) derivative of DC-OPV having two cholesteryl units at the termini as a fluorescent compound. This fluorescent DC-OPV is designed to be accommodated to the G-CLC host of CD8 and 11-BP without their phase separation.

A fluorescent G-CLC is prepared from a dichloromethane solution of CD8, 11-BP and DC-OPV at a weight ratio of 98.4:1.6:1.0, respectively. The solidified G-CLC film is prepared by supercooling from the CLC mesophase temperature to $0{ }^{\circ} \mathrm{C}$. When the G-CLC film is supercooled from the mesophase temperatures at 83,88 and $93^{\circ} \mathrm{C}$, the PBG wavelength shows at 573, 517 and 440, respectively. The PBG wavelength decreases as the annealing temperature increases. The PBG tuning range is relatively wide from 400 to $600 \mathrm{~nm}$. Owing to the formation of a glassy solid-state, the tuned PBG wavelength of G-CLC film remains extremely intact at room temperature even after being stored for 10 months or more.

Thus, this G-CLC film has unique capabilities that not only ensure the fine-tuning of PBG wavelength by annealing temperature, but also allow the on-demand preservation of the shifted PBG wavelength through the subsequent supercooling process. Taking advantage of these salient features, a CG-PBG structure can be readily inscribed inside the G-CLC film by the supercooling process in order to create a new tunable CLC solid-state laser. Figure 9a presents a photograph of the CG-PBG region in a G-CLC film that is solidified by supercooling when the annealing temperature is changed from $85^{\circ} \mathrm{C}$ (right side) to $95^{\circ} \mathrm{C}$ (left side). Interestingly, the reflection color continuously alters from blue to green along the $1 \mathrm{D}$ gradation of annealing temperature of the G-CLC film.

Considering the CG-PGB structure inscribed in the G-CLC film, we then demonstrate the local position-dependent tuning of laser emission. Figure $9 \mathrm{~b}$ shows the microscopic reflection (upper) and optically excited laser emission (lower) spectra of the CG-PGB structure in G-CLC film. We conduct stepwise measurements of microscopic reflection spectra in the CG-PBG region upon moving the G-CLC film with respect to a probing light. The PBG wavelength continuously shifts from 470 to $550 \mathrm{~nm}$, and reflection color is concomitantly changed from blue to green (upper insets in Figure 9b).

As an extension of these findings, we measure the microscopic laser emission spectra in the CG-PBG region. A single laser emission peak is generated by optical excitation with $418 \mathrm{~nm}$ light of the pulse energy of $\sim 500 \mathrm{~nJ}$ per pulse. When the local excitation area in the CG-PBG region is moved in a stepwise manner, the laser emission peak concomitantly shifts in a wide wavelength range from 475 to $550 \mathrm{~nm}$. Furthermore, we observe the changes in microscopic laser emission colors from blue to green (lower insets in Figure 9b). Such salient performances cannot be attained by commercially available CLCs. Taking the overall results into account, the technologically relevant performances might be limited to this kind of chemically designed G-CLCs. Such unique performances cannot be realized by using commercially available CLC materials. ${ }^{17,19,26-39}$ The findings are of interest in the chemical approach to new light-emitting G-CLCs for technological developments for next-generation molecular optoelectronics devices.

\section{CCs AS PCs}

Colloids are generally defined as small particles with a size in the range from a few nanometers to several micrometers. Currently, such

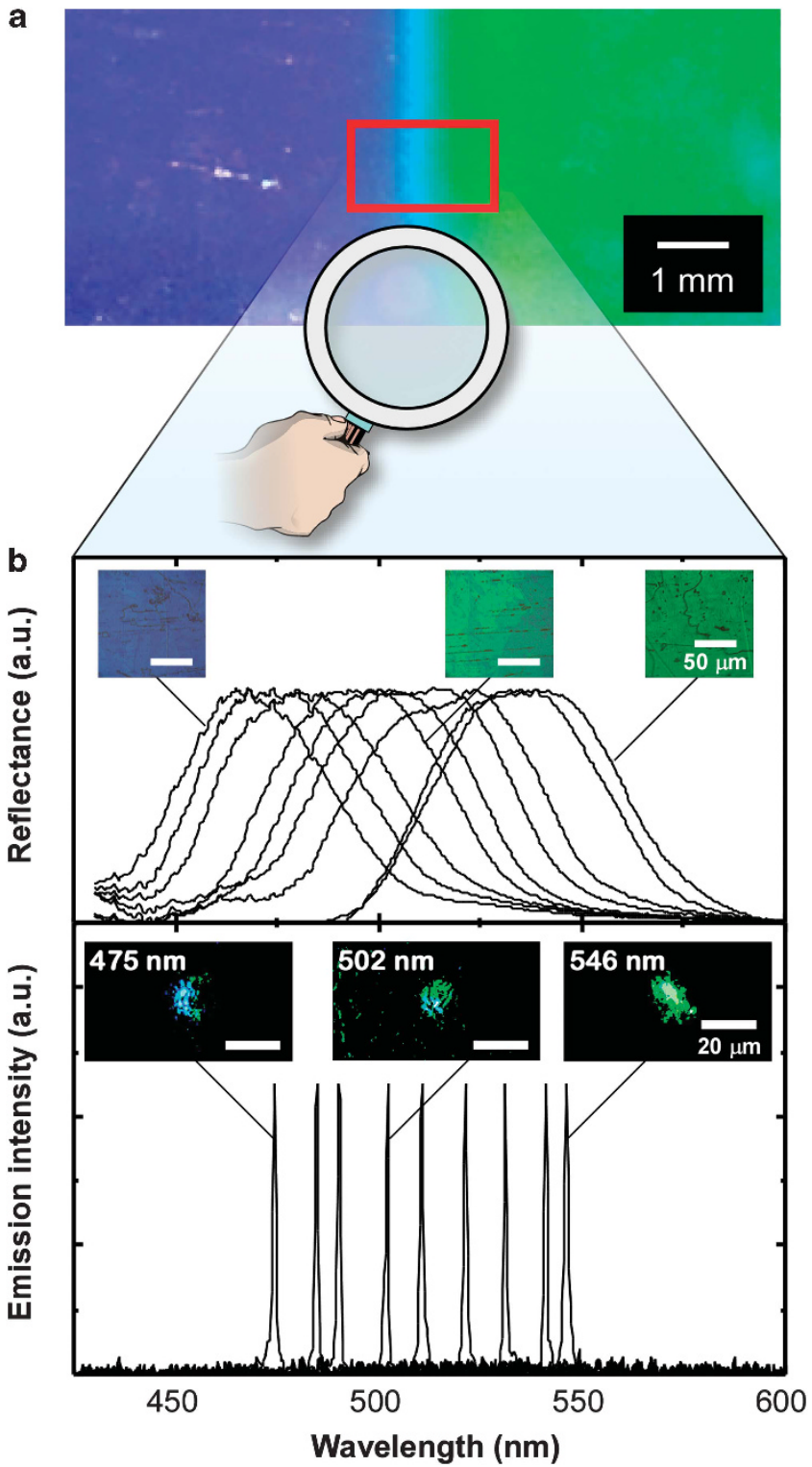

Figure 9 (a) A photograph of a glass-forming chiral liquid crystal (G-CLC) film with continuously-gradated photonic band-gap (CG-PBG) structure. The chemical structures of G-CLC are shown in Figure 8. This G-CLC film was prepared by supercooling from annealing temperature with continuously incremental changes from $85^{\circ} \mathrm{C}$ (right side) to $95^{\circ} \mathrm{C}$ (left side). This photograph was taken at room temperature. (b) The local position-dependent reflection (upper) and optically excited laser emission spectra (lower) of a G-CLC film with CG-PBGs. The spectra were taken upon stepwise translation of the measuring spot. The laser emission spectra were obtained by optical excitation with pulsed $418 \mathrm{~nm}$ light with the energy of $\sim 500 \mathrm{~nJ}$ per pulse. The insets are the microscopic optical images of reflection (upper) and laser emission (lower); the left, middle and right photographs correspond to the images with PBGs centered at 470, 490 and 540, respectively. The scale bars of reflection and laser emission images represent 50 and $20 \mu \mathrm{m}$, respectively. Reproduced with permission from Furumi ${ }^{19}$ and Furumi and Tamaoki. ${ }^{80}$

colloidal microparticles have been commercially available as inks, dry toners, coating materials, spacers for LC displays and biomedical tags in a wide variety of technological applications. From the standpoint of fundamental researches, the microparticles also have been frequently utilized as experimental tools in various research fields, such as 
chemistry, applied physics and biology. Recently, monodispersed microparticles with a diameter of several hundreds of nanometers have garnered considerable attention in the intriguing research field of PCs.

Monodispersed colloidal microparticles with a diameter of several hundred nanometers are known to show the self-assembly from a colloidal suspension into 3D ordered lattice structures (Figure 2b). ${ }^{81-91}$ Such 3D ordered structures of microparticles are called 'CCs'. Notably, no special facilities are required for the fabrication of CC structures due to bottom-up process of microparticle assembly. Therefore, CCs can be prepared with a low cost and on a large scale by the bottom-up process. When the particle diameter is several hundred nanometers, uniform CCs allow us to visualize the PBG as Bragg reflection colors. The maximum reflection wavelength $\left(\lambda_{\max }\right)$ is numerically expressed by Bragg's equation with Snell's law. ${ }^{92}$

$$
\lambda_{\max } \approx 2 d\left(n^{2}-\sin ^{2} \theta\right)^{1 / 2}
$$

where $n$ is the effective refractive index of materials, $d$ is the spacing between lattice planes of CCs and $\theta$ is the angle of incident light. In addition, the effective refractive index $(n)$ is determined by the filling ratio $(f)$ of the microparticles in the CCs as follows.

$$
n \approx\left\{n_{\mathrm{p}}^{2} f+n_{\mathrm{b}}^{2}(1-f)\right\}^{1 / 2}
$$

where $n_{\mathrm{p}}$ and $n_{\mathrm{b}}$ denote the refractive indices of the microparticles and background materials, respectively. These approximate equations can be applied only when the refractive index contrast of materials is not so high. According to the equations (6) and (7), the reflection wavelength can be tuned by controlling the refractive indices, particle radii and filling ratio. ${ }^{81-91}$

The artificial CC structures have been for the first time experimentally prepared by natural sedimentation of colloidal silica microparticles, leading to the extensive development of CCs into the PC research field. ${ }^{92}$ To date, much effort has established prevalent guidelines for fabricating high-quality CC films by vertical deposition of a substrate in a suspension, lifting of a substrate from a suspension, shearing of a suspension between substrates, and so forth. ${ }^{81-91}$ With respect to photonic device applications, the recent CC research advances have been directed toward the development of an extensive variety of optical reflection sensors and displays that are capable of shifting the reflection wavelength, that is PBG wavelength, by changes in external stimuli, such as the temperature, ionic strength, $\mathrm{pH}$, photoirradiation, and electric and magnetic fields. ${ }^{81-91}$ However, the previous reports have been made on the fabrication of optical reflection devices by utilizing passively the reflection properties of CC films.

Recently, we developed new potential uses for high-quality CCs in flexible and tunable polymer laser devices by low-threshold optical excitation. ${ }^{93-95}$ These applications are realized by utilizing actively the reflection properties of CC films. The following sections present not only the fascinating studies with CCs reported by other research groups, but also some of our own experimental results.

\section{FLEXIBLE LASER ACTION FROM POLYMER CCS}

Significant progress in PC researches has promoted the manipulation of photons from light-emitting organic and polymer materials within a small space. In particular, the modified spontaneous emission and stimulated emission from organic and polymer PCs provide new opportunities for their use in low-threshold lasers, highly efficient light-emitting devices, and so forth. As organic and polymer materials have become technologically important because of their flexible properties for device fabrication and their tunabilities of optoelectronic properties depending on the molecular structures.

Recently, we succeeded in obtaining optically excited laser action from high-quality CC films composed of polymer materials. ${ }^{93}$ The most interesting performance is the lasing action even with a bent shape of the device. As illustrated in Figure 10, we design and prepare a CC-laser (CC-L) cavity structure. In this CC-L cavity, a lightemitting planar defect is embedded between a pair of polymer CC films. In order to prepare the light-emitting planar defect, we use Rhodamine 640 dye $(\mathrm{Rh})$ as a fluorescent material, a relatively low molecular weight poly(ethylene glycol) diacrylate as a matrix, and 2-benzyl-2-(dimethylamino)-4'-morpholinobutyrophenone as a photopolymerisation initiator. A CC film of polystyrene microparticles with a diameter of $200 \mathrm{~nm}$, which is substantially stabilized by a poly(dimethylsiloxane) (PDMS) matrix, is prepared by the natural drying technique with silicone oil. ${ }^{96}$ After a mixture of $\mathrm{Rh}$, poly(ethylene glycol) diacrylate and 2-benzyl-2-(dimethylamino)-4'morpholinobutyrophenone is injected between a pair of the CC films, a durable CC-L cavity can be obtained by photopolymerisation of poly(ethylene glycol) diacrylate used in the light-emitting planar defect. Indeed, the microscopic structure of CC-L cavity containing a light-emitting planar defect with a thickness of $\sim 2.8 \mu \mathrm{m}$ is observed by scanning electron microscopy. from the cross-sectional direction (right picture in Figure 10).

The reflection and emission spectral results of the CC-L cavity are shown in Figures 11a and b. The CC-L cavity shows a characteristic reflection band in a wavelength from 600 to $630 \mathrm{~nm}$, originating from the PGB of CC film (Figure 11a). Subsequently, the laser emission properties are investigated by optical excitation with pulsed $532 \mathrm{~nm}$ light. When the optical excitation energy is set at a low value of $\sim 70 \mathrm{~nJ}$ per pulse, the emission spectrum shows partial modification in a broad emission band of Rh (blue curve in Figure 11b). The emission is distinctly forbidden in a wavelength range from 600 to $630 \mathrm{~nm}$ by the PBG of the CC film used in the CC-L cavity. When the excitation energy successively increases, the broad emission spectrum is suddenly changed to be the laser emission at the energy of $\sim 210 \mathrm{~nJ}$ per pulse (red curve in Figure 11b). At this excitation energy, a single laser emission peak appears at $\sim 611 \mathrm{~nm}$. The threshold excitation energy for the laser-feedback effect is $130 \mathrm{~nJ}$ per pulse. In this experiment, the pulse duration and focused diameter of the excitation beam are set at $3 \mathrm{~ns}$ and $200 \mu \mathrm{m}$, respectively. Therefore, the lasingthreshold excitation peak power is calculated to be $138 \mathrm{~kW} \mathrm{~cm}^{-2}$. From exploring the precedents of lasing from various CCs, this threshold power is two orders of magnitude lower than that of the previously investigated CCs. ${ }^{97-101}$ This experimental fact implies that the emitting photons from the planar defect is effectively confined in the orthogonal direction of the CC-L cavity, leading to the lowthreshold laser action.

The lasing mechanism from this CC-L cavity can be rationalized in terms of the defect-mode localized in the PBG of the CC film. Such defect-mode lasing is definitely consistent with several precedents for 1D-PCs, such as dielectric multilayered mirrors and cholesteric LCs, with light-emitting planar defects. ${ }^{102,103}$ In this CC-L cavity, the theoretical wavelength of the defect-mode can be calculated on the basis of the scalar-wave approximation ${ }^{104}$ results of the CC film. As a result, the theoretical defect-mode wavelength is found to be $613 \mathrm{~nm}$ (gray arrow in Figure 11b). This calculated wavelength is in fairly good agreement with the single laser emission peak observed for the CC-L cavity. The inset of Figure 11b shows the high-resolution laser emission spectrum of the CC-L cavity. At this lasing stage, the emission spectrum linewidth is as narrow as $0.17 \mathrm{~nm}$, and is relatively 
Optical excitation

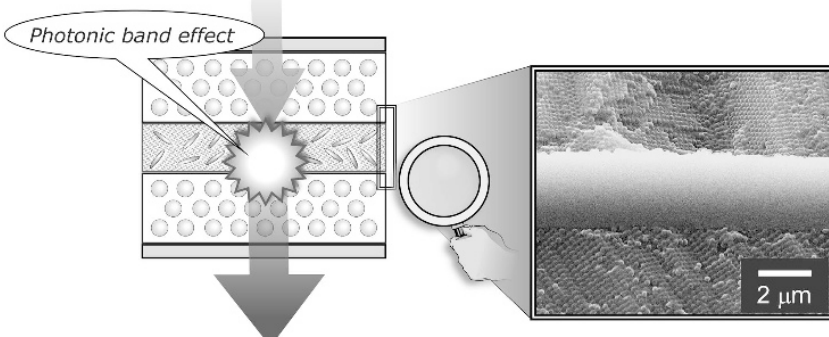

Efficient Laser Action

$\begin{array}{lll}\text { Polystyrene (PS) particle } & \text { Fluorescent rhodamine (Rh) dye } \\ \square \text { Poly(dimethyl siloxane) (PDMS) } & \text { Poly(ethylene glycol) diacrylate (PEG-DA) }\end{array}$

Figure 10 Schematic illustration of a polymer colloidal crystal-laser (CC-L) cavity for a flexible laser device. A light-emitting planar defect containing a fluorescent Rh dye is sandwiched between a pair of CC films of polystyrene particles stabilized by an elastic PDMS matrix. The right-hand picture is a cross-sectional scanning electron microscopy image of the magnified lightemitting planar defect between the CC films. The scale bar in this scanning electron microscopy image represents $2 \mu \mathrm{m}$. Reproduced with permission from Furumi et al. ${ }^{87}$ and Furumi et al. ${ }^{93}$

narrow compared with that for the other CCs reported to date. ${ }^{97-101}$ Such a single and narrow lasing peak is achieved by fabricating a high-quality CC-L cavity, as presented in Figure 10.

As an expansion of these findings, the excellent flexibility of CC-L cavities is accomplished by fabricating them completely with polymer materials. A CC film of polystyrene microparticles stabilized by PDMS can be prepared on a poly(ethylene terephthalate) sheet (inset in Figure 11c). This situation arouses the experimental demonstration of laser action when the CC-L cavity is bent shape by mechanical stress. As can be seen in Figure 11c, the optically excited lasing action can be generated from the polymer CC-L cavity even with the bent shape. Such performance cannot be attained by the laser cavities fabricated with inorganic materials. From the viewpoint of nextgeneration optoelectronics, it would be greatly advantageous to be able to fabricate all-polymer laser devices with such intrinsic properties as flexibility, easy processability, ultra-light weight and low cost. Furthermore, we successfully demonstrated the reversible photoswitching of micropatterned lasing in CCs by the photochromic reaction. ${ }^{105}$

\section{WIDELY TUNABLE LASER ACTION FROM CC GELS}

The reflection wavelength of a CC film is crucially dependent on not only the microparticle diameter, but also the refractive indices and particle distances. Although we can envisage that it is not easy to control and change the refractive indices of materials by external stimuli, the combination of CCs with polymer hydrogels does allow for easy changes in the distance between the colloidal microparticles by swelling or shrinking of the polymer gel networks by absorbing or expelling large amounts of water, respectively. As a result, it is possible to tune the reflection wavelength of CCs with hydrogels in response to changes in the external temperature, $\mathrm{pH}$, target molecules and ions and so forth. ${ }^{81-91}$ To date, numerous effort has been devoted to the development of optical reflection sensors and displays by using the CC hydrogels. However, the polymer hydrogels are quite vulnerable
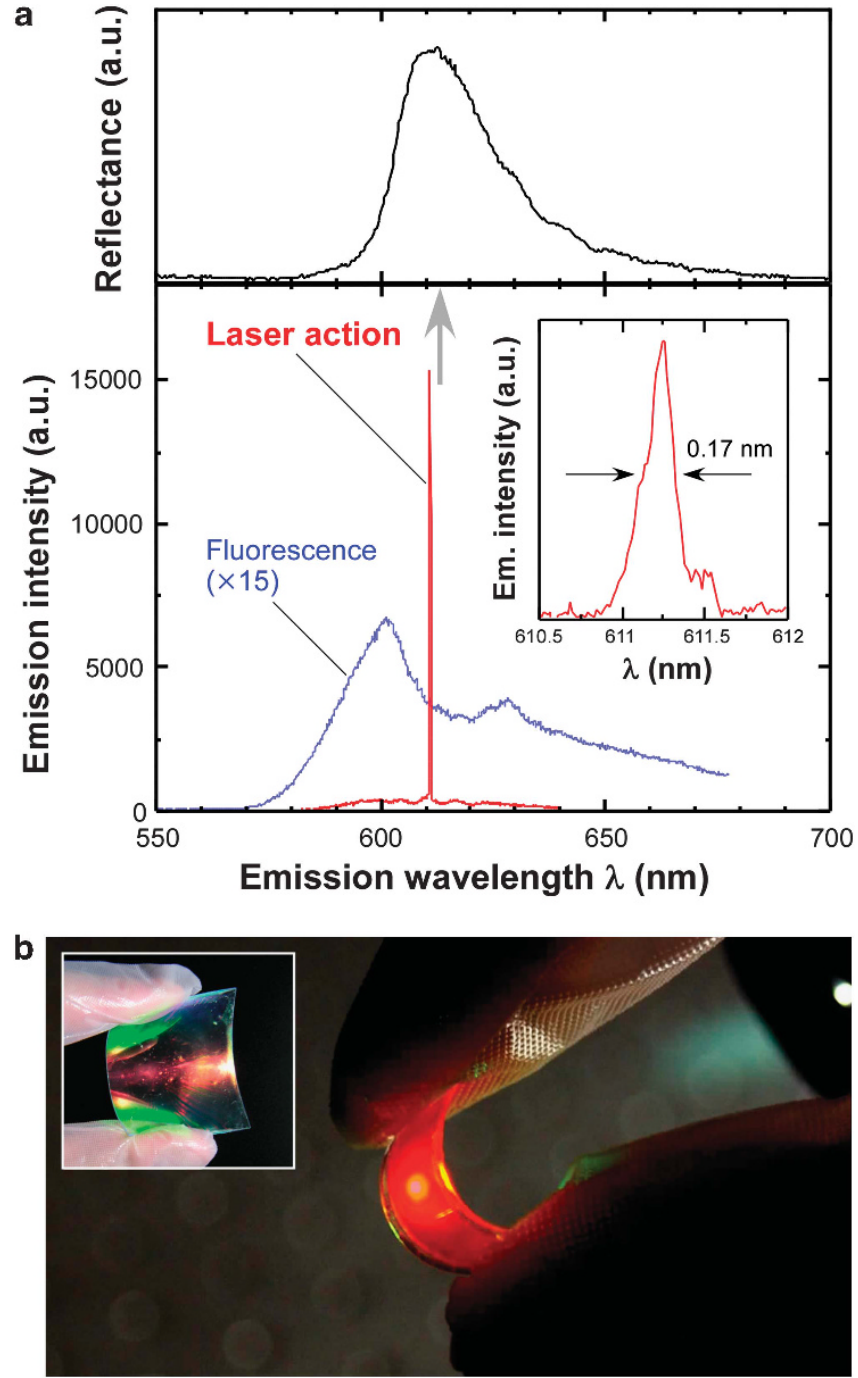

Figure 11 (a) Reflection spectrum of a colloidal crystal-laser (CC-L) cavity with a light-emitting planar defect between a pair of CC films of polystyrene particles stabilized by an elastic PDMS matrix. The cavity structure is presented in Figure 10 (b) Emission spectra of the CC-L cavity by optical excitation with $532 \mathrm{~nm}$ light from an $\mathrm{Nd}$ :yttrium aluminum garnet laser beam with the energies of $\sim 70 \mathrm{~nJ}$ per pulse (blue curve) and $\sim 210 \mathrm{~nJ}$ per pulse (red curve). The intensity of emission spectrum by optical excitation with $\sim 70 \mathrm{~nJ}$ per pulse is magnified 15 times to confirm the spectrum shape (blue curve). The gray arrow represents the theoretical defect-mode wavelength calculated on the basis of the scalar-wave approximation results of the CC film. The inset of this figure shows the high-resolution emission spectrum around $611 \mathrm{~nm}$ during the laser action. (c) A photograph of the optically excited laser action from a CC-L cavity fabricated with all-polymer materials. As evident from this picture, it is possible to demonstrate the optically excited laser action when the polymer CC- $L$ cavity is bent under mechanical stress. The inset shows a photograph of a flexible CC film formed on a poly(ethylene terephthalate) sheet. Reproduced with permission from Furumi et al. ${ }^{87}$ and Furumi et al. ${ }^{93}$

under dry atmospheric conditions, wherein their physical properties, such as toughness and elasticity, completely vanish.

To address this issue, we prepare a permanently stable CC gel film for tunable laser applications by using a non-volatile room-temperature ionic liquid. ${ }^{94}$ Figure 12 depicts the research concept of widely tunable lasing from a CC-L gel cavity by applying mechanical stress. Unlike the CC-L cavities described above (Figure 10), this CC-L gel 


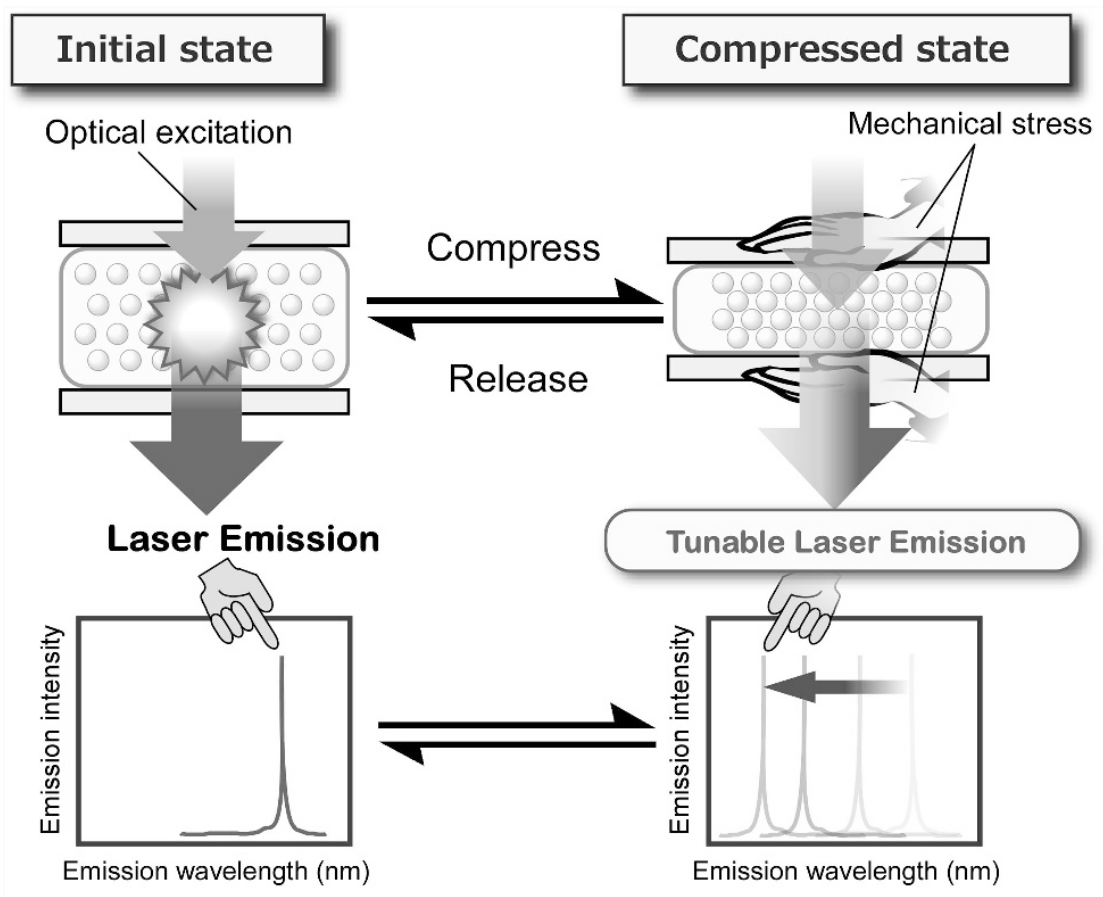

PS particle (Diameter: $120 \mathrm{~nm}$ )

Polymer Gel with fluorescent dye in RIL

Figure 12 Schematic illustration of mechanical tunable laser from in a colloidal crystal-laser (CC-L) gel cavity. This CC-L gel cavity is a non-close-packed colloidal crystal (CC) of polystyrene (PS) microparticles, which is embedded in a polymer gel with a room-temperature ionic liquid (RIL) solution of fluorescent organic dye(s). When mechanical stress is applied to the CC-L gel cavity, the single laser emission peak can be tuned over a wide wavelength range. Reproduced with permission from Furumi et al. ${ }^{94}$

cavity does not contain a light-emitting planar defect between a pair of CC films. This structure is very simple. The CC-L gel cavity is made of a non-close-packed CC gel film of polystyrene microparticles with a diameter of $120 \mathrm{~nm}$, fabricated by the shearing technique of the colloidal suspension ${ }^{106-108}$ and stabilized by an room-temperature ionic liquid solution of fluorescent organic dye(s). We use poly $(\mathrm{N}$ methylolacrylamide-co- $N, N^{\prime}$-methylenebisacrylamide) as a thermoinsensitive polymer hydrogel and 1-allyl-3-butylimidazolium bromide (ABImBr) as an room-temperature ionic liquid. Importantly, $\mathrm{ABImBr}$ shows good miscibility with water, thereby enabling a swelling state of the polymer gel in this room-temperature ionic liquid. Therefore, the CC gel film can be permanently stabilized by $\mathrm{ABImBr}$ even in dry atmospheres. ${ }^{109}$ As fluorescent dyes, we use $\mathrm{Rh}$ and sulforhodamine B (SR), whose chemical structures are depicted in Figure 13a.

The as-prepared CC-L gel cavity containing an $\mathrm{ABImBr}$ solution of $\mathrm{Rh}$, that is, $\mathrm{Rh} / \mathrm{ABImBr}$, shows a reflection band centred at $690 \mathrm{~nm}$. When the CC-L gel cavity is compressed by mechanical stress in the film thickness direction, the reflection band shifts to the shorter wavelength due to the geometric decrease in the distance between the lattice planes of CCs. By applying stress at a compression ratio of $\sim 10 \%$, the reflection band blue-shifts from 690 to $645 \mathrm{~nm}$ (gray curve in Figure 13b). This reflection band is wholly covered by the broad fluorescence spectrum of $\mathrm{Rh} / \mathrm{ABImBr}$ solution with the maximum fluorescence wavelength at $610 \mathrm{~nm}$ (dashed curve in Figure 14a). Thus, it is anticipated that the laser-feedback effect is efficiently generated by the PBG effect of CC-L gel cavity.
Subsequently, the laser emission characteristics of this CC-L gel cavity are evaluated by optical excitation with second harmonic generation light at $532 \mathrm{~nm}$ from a Nd:yttrium aluminum garnet laser beam. Figures $13 \mathrm{~b}$ and $\mathrm{c}$ show the emission spectra of the CC-L gel cavity obtained by optical excitation below and above the energy required for the laser-feedback effect, respectively. From a relatively low excitation energy up to $\sim 100 \mathrm{~nJ}$ per pulse, the emission intensity monotonously is intensified according to the excitation energy. When the excitation energy is set at $120 \mathrm{~nJ}$ per pulse, we observe the following extraordinary modification in a broad fluorescence spectrum of Rh (black curve in Figure 13b). The emission spectrum is inhibited in a wavelength range of the reflection band. Most importantly, the emission spectrum shows drastic increase in emission intensity near the longer-wavelength reflection band edge. This finding is of paramount importance. Such emission spectral change occurs due to the resonant enhancement of the density of state of the emitting photons near the reflection band edge of the CC-L gel cavity. This experimental fact provides clear evidence that the reflection band of CC-L gel cavity works as the PBG.

When the excitation energy exceeds $330 \mathrm{~nJ}$ per pulse, a single laser emission peak is observed at $653 \mathrm{~nm}$ (black curve in Figure 13c). At the lasing stage, the emission spectral linewidth is as narrow as $0.06 \mathrm{~nm}$. To our knowledge, this emission spectral linewidth is narrowest as compared with those for previously reported CC-L cavities. ${ }^{97-102}$ The single lasing peak appears near the longerwavelength edge of reflection band due to a strong localization of emitting photons from $\mathrm{Rh}$ in the high optical quality CC-L gel 


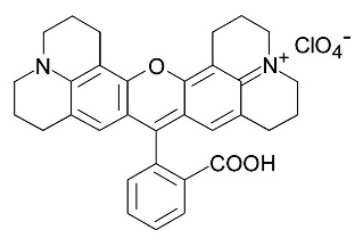

Rh

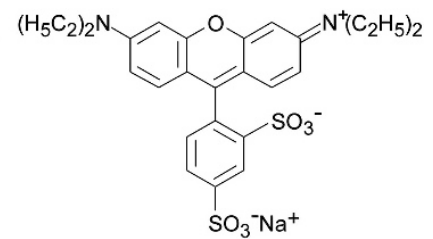

SR b

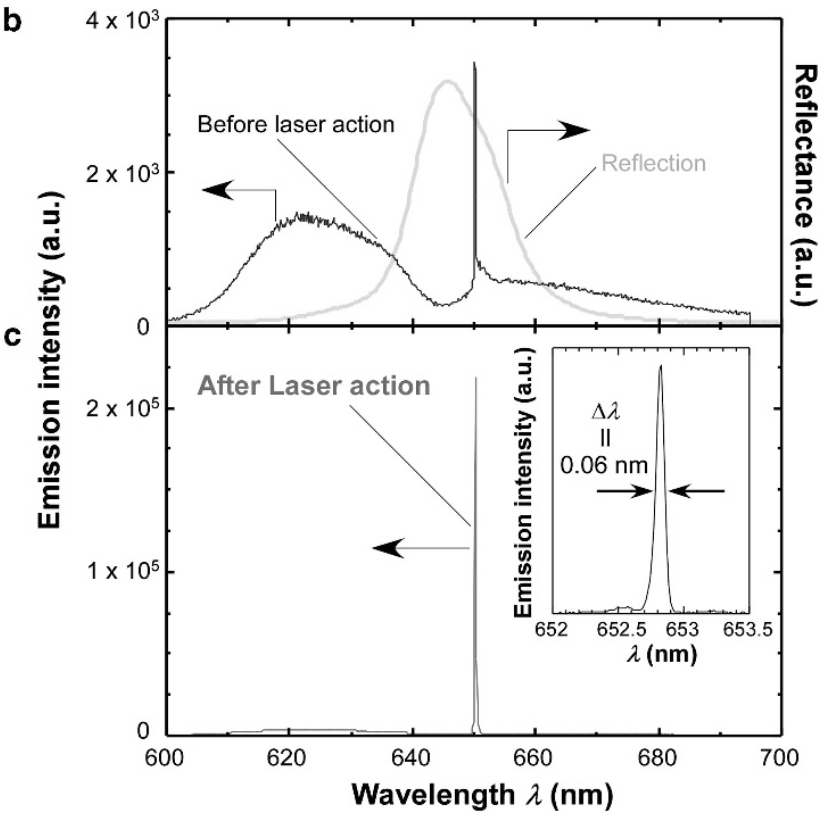

Figure 13 (a) Chemical structures of the fluorescent dyes of Rhodamine $640(\mathrm{Rh})$ and sulforhodamine B (SR). (b) Reflection (gray curve) and emission (black curve) spectra of a colloidal crystal-laser (CC-L) gel cavity with an ionic liquid solution of Rh. As illustrated in Figure 12, the reflection wavelength of the CC-L gel cavity was tuned at $645 \mathrm{~nm}$ by applying mechanical stress at a compression ratio of $\sim 10 \%$ in the film thickness direction. The broad emission spectrum was measured just before the laser action when the excitation energy was set at $120 \mathrm{~nJ}$ per pulse. (c) Laser emission spectrum (red curve) from the CC-L gel cavity with an Rh solution by optical excitation with pulsed $532 \mathrm{~nm}$ light at the energy of $330 \mathrm{~nJ}$ per pulse. The inset of this figure shows the high-resolution emission spectrum around $653 \mathrm{~nm}$. Reproduced with permission from Furumi et al. ${ }^{94}$

cavity. ${ }^{110,111}$ Furthermore, the single lasing peak emerges only at the longer-wavelength edge, but not at the shorter-wavelength edge of reflection band. This phenomenon can be interpreted by considering the self-absorption of $\mathrm{Rh}$ at the shorter wavelength of reflection band due to relatively high concentration of the $\mathrm{Rh} / \mathrm{ABImBr}$ solution. To date, there has been one similar precedent for optically excited laser action from a CC-L cavity of fluorescent mesoporous silica microparticles stabilized by polyacrylamide. ${ }^{100}$ However, the laser action mechanism still remains obscure from the viewpoint of the PBG effect. Taking account of our overall experimental results, it is plausible that a single lasing peak from the CC-L gel cavity emerges due to the PBG band-edge effect resulting from the density of state enhancement of the emitting photons.

On account of the intrinsic property of the CC-L gel cavity-that the reflection wavelength can be tuned by applying mechanical stress-we then demonstrate the mechanical tuning of the laser emission peak upon applying compression stress. Figure 14a shows

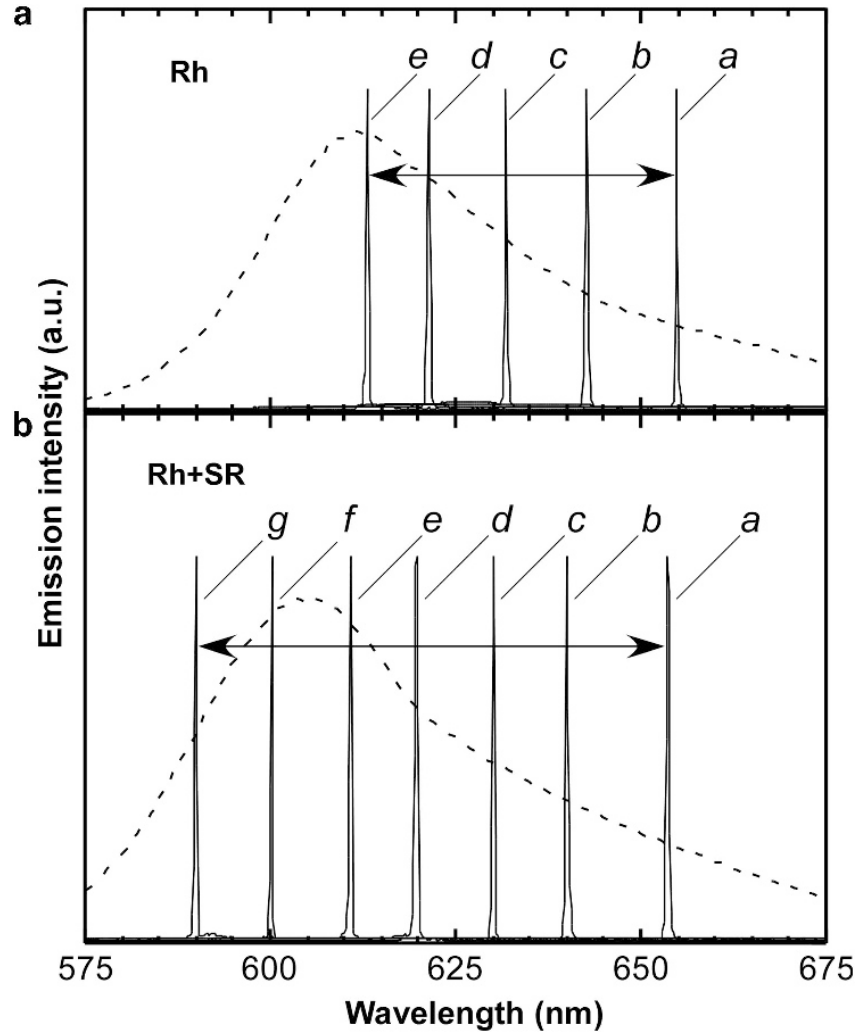

Figure 14 Changes in laser emission spectra of colloidal crystal-laser (CC-L) gel cavities with RIL solutions of Rh (a) and Rh+ SR (b) by compression with mechanical stress, as presented in Figure 12. Each CC-L gel cavity was continuously compressed at 9\% (curve 1), 12\% (curve 2), 15\% (curve 3), $18 \%$ (curve 4 ), $21 \%$ (curve 5), 22\% (curve 6), and 25\% (curve 7 ) in the film thickness direction. The dashed curves indicate the broad-band fluorescence spectra of $\mathrm{Rh}$ and $\mathrm{Rh}+\mathrm{SR}$ solutions by optical excitation with $532 \mathrm{~nm}$ light from a Xe lamp. Reproduced with permission from Furumi et al. ${ }^{94}$

the changes in laser emission spectra of the CC-L gel cavity with an $\mathrm{Rh} / \mathrm{ABImBr}$ solution as a function of the compression ratio of film thickness. When this CC-L gel cavity is compressed by mechanical stress in a stepwise way between a compression ratio from 9 to $21 \%$, the laser emission peak continuously shifts from 655 to $612 \mathrm{~nm}$ due to the changes in PBG wavelength. Importantly, the single laser emission peak can be preserved even with a compressed state. Except for this wavelength range, it is impossible to generate the laser action. The optical gain spectrum typically shows a similar spectral appearance as the fluorescence spectrum. Therefore, the single laser emission peak appears when the reflection band overlapped with a fluorescence band of $\mathrm{Rh}$ (dashed curve in Figure 14a). The tuning range of laser emission peak is $43 \mathrm{~nm}$ for this CC-L gel cavity with $\mathrm{Rh} / \mathrm{ABImBr}$. This tuning range is not sufficient.

Further expansion of the laser tuning wavelength range can be realized by an $A B I m B r$ solution of a mixture of $\mathrm{Rh}$ and $\mathrm{SR}$, that is $\mathrm{Rh}+\mathrm{SR} / \mathrm{ABImBr}$ (Figure $14 \mathrm{~b}$ ). The appropriate addition of SR to $\mathrm{Rh}$ leads to a broad fluorescence spectrum centered at $604 \mathrm{~nm}$ through the energy transfer (dashed curve in Figure 14b). Figure 14b shows the mechanical tuning result of laser emission peak from a CC-L gel cavity with an $\mathrm{Rh}+\mathrm{SR} / \mathrm{ABImBr}$ solution. Mechanical compression of this CC-L gel cavity enables the continuous tuning of a single laser 
emission peak from 655 to $588 \mathrm{~nm}$. This tuning range for the laser emission peak is relatively wide, compared with those in previous reports of mechanically tunable laser emission from the various laser cavity structures fabricated by elastic polymers. ${ }^{98,112-114}$

The CC-L gel cavity, shown here, consists of an ordered CC structure of polystyrene microparticles stabilized by a polymer gel and ionic liquid. Therefore, the ionic liquid solution of fluorescent organic dyes in the CC-L gel cavity can be freshly replaced through the circulation using a procedure similar to that adopted in conventional dye lasers. Such technologically relevant performances, such as mechanical tuning of laser emission peaks and replacement of fluorescent dye solutions, might be limited to this kind of CC-L gel cavity with an ionic liquid.

\section{CONCLUSIONS}

This article presented an overview of self-assembled PCs of organic and polymer materials such as CLCs and CCs for a wide variety of laser applications. Recently, the CLCs and CCs as tools for fabricating the PCs have attracted interest from fundamental and technological viewpoints. This is because both CLC molecules and colloidal microparticles can spontaneously organize $1 \mathrm{D}-\mathrm{PC}$ and $3 \mathrm{D}-\mathrm{PC}$ structures with PBGs in a visible wavelength range, respectively. Stimulated laser emission from CLCs or CCs doped with a fluorescent dye can be generated by optical excitation. The laser emission peak takes place at the PBG edge(s) or in the PBG wavelength range due to the internal laser-feedback effect.

For dye-doped CLC cells, the optically excited laser emission shows the circular polarization characteristic even though the excitation light is linearly polarized. Applying certain voltages to optically excited CLC cells brings about reversible switching of the laser emission peak. Moreover, the laser emission peak can be tuned by using photoreactive CLCs through their photochemical reactions. Recently, much effort has targeted on the position-dependent tuning of laser emission peaks by CG-PBG of CLCs. Appropriate molecular design and synthesis of CLCs are of paramount importance in the fabrication of stable CG-PBG structures of fluorescent G-CLC oligomers. Continuously and reversibly tunable laser action from the G-CLC cell can be achieved by low-threshold optical excitation. With regard to CCs, we design and fabricate a laser cavity structure consisting of a light-emitting planar defect between a pair of polymer CC films. The low-threshold lasing action is demonstrated by successful confinement of emitting photons in the laser cavity. When the laser cavity is fabricated with all-polymer materials, it is possible to generate optically excited laser action even with a bent shape of the laser cavity. Moreover, we succeed in the widely tunable lasing from CC gel films permanently stabilized by an ionic liquid.

Researches with CLCs and CCs should aid the development of next-generation miniaturized photonics devices. We believe that tailoring the molecular design of CLCs and CCs and their laser cavity structures enables a variety of applications in next-generation optoelectronic devices of organic and polymer materials.

\section{CONFLICT OF INTEREST}

The author declares no conflict of interest.

\section{ACKNOWLEDGEMENTS}

SF would like to thank Professor S Yokoyama of the Kyushu University, Drs A Otomo and S Mashiko of the National Institute of Information and Communications Technology (NICT), and Professor N Tamaoki of the Hokkaido University for their technical advices and helpful discussions on CLCs. SF is also indebted to Drs T Sawada, HT Miyazaki, H Fudouzi,
M Nishino and Y Sakka of the NIMS and Professor. T Kanai of the Yokohama National University for their valuable suggestion on the CCs. This work was supported in part by the Precursory Research for Embryonic Science and Technology (PRESTO) project of the Japan Science and Technology Agency (JST), the Strategic Information and Communications R\&D Promotion Programme (SCOPE) project from the Ministry of Internal Affairs and Communications (MIC), the Grant-in-Aid for Young Scientist (A) from the Ministry of Education, Science, Sports and Culture (MEXT), the Kao Foundation for Arts and Sciences, and the Yazaki Memorial Foundation for Science \& Technology.

1 López, C. Materials aspects of photonic crystals. Adv. Mater. 15, 1679-1704 (2003).

2 Yablonovitch, E. Inhibited spontaneous emission in solid-state physics and electronics. Phys. Rev. Lett. 58, 2059-2062 (1987).

3 John, S. Strong localization of photons in certain disordered superlattices. Phys. Rev. Lett. 58, 2486-2489 (1987).

4 Ohtaka, K. Energy-band of photons and low-energy photon diffraction. Phys. Rev. B 19, 5057-5067 (1979)

5 Joannopoulos, J. D., Meade, R. D. \& Winn, J. N. Photonic Crystals: Molding the Flow of Light 3-5 (Princeton University Press, Princeton, 1995).

6 Painter, O., Lee, R. K., Scherer, A., Yariv, A., O'Brien, J. D., Dapkus, P. D. \& Kim, I. Two-dimensional photonic band-gap defect mode laser. Science 284, 1819-1821 (1999).

7 Noda, S., Chutinan, A. \& Imada, M. Trapping and emission of photons by a single defect in a photonic bandgap structure. Nature 407, 608-610 (2000).

8 Akahane, Y., Asano, T., Song, B.-S. \& Noda, S. High-Q photonic nanocavity in a twodimensional photonic crystal. Nature 425, 944-947 (2003).

9 Inoue, K., Wada, M., Sakoda, K., Yamanaka, A., Hayashi, M. \& Haus, J. W. Fabrication of 2-dimensional photonic band-structure with near-infrared band-gap. Jpn J. Appl. Phys. 33, L1463-L1465 (1994).

10 Rosenberg, A., Tonucci, R. J. \& Bolden, E. A. Photonic band-structure effects in the visible and near ultraviolet observed in solid-state dielectric arrays. Appl. Phys. Lett. 69, 2638-2640 (1996).

11 Masuda, H., Ohya, M., Asoh, H., Nakao, M., Nohtomi, M. \& Tamamura, T. Photonic crystal using anodic porous alumina. Jpn. J. Appl. Phys. 38, L1403-L1405 (1999).

12 Lin, S. Y., Fleming, J. G., Hetherington, D. L., Smith, B. K., Biswas, R., Ho, K. M., Sigalas, M. M., Zubrzycki, W., Kurtz, S. R. \& Bur, J. A three-dimensional photonic crystal operating at infrared wavelengths. Nature 394, 251-253 (1998).

13 Noda, S., Tomoda, K., Yamamoto, N. \& Chutinan, A. Full three-dimensional photonic bandgap crystals at near-infrared wavelengths. Science 289, 604-606 (2000).

14 Sun, H. B., Matsuo, S. \& Misawa, H. Three-dimensional photonic crystal structures achieved with two-photon-absorption photopolymerization of resin. Appl. Phys. Lett. 74, 786-789 (1999).

15 Deubel, M., Freymann, G. V., Wegener, M., Pereira, S., Busch, K. \& Soukoulis, C. M. Direct laser writing of three-dimensional photonic-crystal templates for telecommunications. Nature Mater. 3, 444-447 (2004).

16 Campbell, M., Sharp, D. N., Harrison, M. T., Denning, R. G. \& Turberfield, A. J. Fabrication of photonic crystals for the visible spectrum by holographic lithography. Nature 404, 53-56 (2000).

17 Ford, A. D., Morris, S. M. \& Coles, H. J. Photonics and lasing in liquid crystals. Mater. Today 9, 36-42 (2006).

18 Coles, H. in Handbook of Liquid Crystals, vol. 2A, (eds Demus, D., Goodby, J., Gray, G. W., Spiess, H.-W. \& Vill, V) Ch. IV-2 335-409 (Wiley-VCH, Weinheim, 1998).

19 Furumi, S. Recent progress in chiral photonic band-gap liquid crystals for laser applications. Chem. Rec. 10, 394-408 (2010).

20 Broer, D. J., Lub, J. \& Mol, G. N. Wide-band reflective polarizers from cholesteric polymer networks with a pitch gradient. Nature 378, 467-469 (1995).

21 Tamaoki, N. Cholesteric liquid crystals for color information technology. Adv. Mater. 13, 1135-1147 (2001)

22 Mallia, V. A. \& Tamaoki, N. Design of chiral dimesogens containing cholesteryl groups; formation of new molecular organizations and their application to molecular photonics. Chem. Soc. Rev. 33, 76-84 (2004).

23 Akagi, K. \& Mori, T. Helical polyacetylene-origins and synthesis. Chem. Rec. 8, 395-406 (2008).

24 Nishiyama, I. Remarkable effect of pre-organization on the self assembly in chiral liquid crystals. Chem. Rec. 9, 340-355 (2009).

25 O'Neill, M. \& Kelly, S. M. Liquid crystals for charge transport, luminescence, and photonics. Adv. Mater. 14, 1135-1146 (2003).

26 Ozaki, M., Matsuhisa, Y., Yoshida, H., Ozaki, R. \& Fujii, A. Photonic crystals based on chiral liquid crystal. phys. stat. sol. (a) 204, 3777-3789 (2007).

27 Graham-Rowe, D. A new twist to tuning lasers. Nature Photon 3, 182-183 (2009).

28 Coles, H. \& Morris, S. Liquid-crystal laser. Nature Photon 4, 676-685 (2010).

29 Goldberg, L. S. \& Schnur, J. M. Tunable internal-feedback liquid crystal-dye laser. US Patent 3771065 (1973).

30 Kopp, V. I., Fan, B., Vithana, H. K. M. \& Genack, A. Z. Low-threshold lasing at the edge of a photonic stop band in cholesteric liquid crystals. Opt. Lett. 23, 1707-1709 (1998) 
31 Munoz, A., Palffy-Muhoray, P. \& Taheri, B. Ultraviolet lasing in cholesteric liquid crystals. Opt. Lett. 28, 804-806 (2001).

32 Amemiya, K., Shin, K.-C., Takanishi, Y., Ishikawa, K., Azumi, R. \& Takezoe, H. Lasing in cholesteric liquid crystals doped with oligothiophene derivatives. Jpn. J. Appl. Phys. 43, 6084-6087 (2004).

33 Watanabe, Y., Uchimura, M., Araoka, F., Konishi, G., Watanabe, J. \& Takezoe, H. Extremely low threshold in a pyrene-doped distributed feedback cholesteric liquid crystal laser. Appl. Phys. Exp. 2, 102501 (2009).

34 Ozaki, M., Kasano, M., Ganzke, D., Hasse, W. \& Yoshino, K. Mirrorless lasing in a dyedoped ferroelectric liquid crystal. Adv. Mater. 14, 306-309 (2002).

35 Finkelmann, H., Kim, S. T., Munoz, A., Palffy-Muhoray, P. \& Taheri, B. Tunable mirrorless lasing in cholesteric liquid crystalline elastomers. Adv. Mater. 13, 10691072 (2001).

36 Schmidtke, J., Stille, W., Finkelmann, H. \& Kim, S. T. Laser emission in a dye doped cholesteric polymer network. Adv. Mater. 14, 746-749 (2002)

37 Matsui, T., Ozaki, R., Funamoto, K., Ozaki, M. \& Yoshino, K. Flexible mirrorless laser based on a free-standing film of photopolymerized cholesteric liquid crystal. Appl. Phys. Lett. 81, 3741-3743 (2002).

38 Schmidtke, J., Kniesel, S. \& Finkelmann, H. Probing the photonic properties of a cholesteric elastomer under biaxial stress. Macromolecules 38, 1357-1363 (2005).

39 Shibaev, P. V., Tang, K., Genack, A. Z., Kopp, V. \& Green, M. M. Lasing from a stiff chain polymeric lyotropic cholesteric liquid crystal. Macromolecules 35, 3022-3025 (2002).

40 Chandrasekhar, S. Liquid Crystals. 2nd edn 292-296 (Cambridge University Press, Cambridge, 1992)

41 Cao, W., Munoz, A., Palffy-Muhoray, P. \& Taheri, B. Lasing in a three-dimensional photonic crystal of the liquid crystal blue phase II. Nature Mater. 1, 111-113 (2002).

42 Yokoyama, S., Mashiko, S., Kikuchi, H., Uchida, K. \& Nagamura, T. Laser emission from a polymer-stabilized liquid-crystalline blue phase. Adv. Mater. 18, 48-51 (2006).

43 Furumi, S., Yokoyama, S., Otomo, A. \& Mashiko, S. Electrical control of the structure and lasing in chiral photonic band-gap liquid crystals. Appl. Phys. Lett. 82, 16-18 (2003).

44 Solladié, G. \& Zimmermann, R. G. Liquid-crystals: A tool for studies on chirality. Angew. Chem. Int. Ed. Engl. 23, 348-362 (1984).

45 Langeveld-Voss, B. M. W., Janssen, R. A. J., Christiaans, M. P. T., Meskers, S. C. J., Dekkers, H. P. J. M. \& Meijer, E. W. Circular dichroism and circular polarization of photoluminescence of highly ordered poly\{3,4-di[(S)-2-methylbutoxy]thiophene\}. J. Am. Chem. Soc. 118, 4908-4909 (1996).

46 Peeters, E., Christiaans, M. P. T., Janssen, R. A. J., Schoo, H. F. M., Dekkers, H. P. J. M. \& Meijer, E. W. Circularly polarized electroluminescence from a polymer lightemitting diode. J. Am. Chem. Soc. 119, 9909-9910 (1997).

47 Oda, M., Nothofer, H.-G., Lieser, G., Scherf, U., Meskers, S. C. J. \& Neher, D. Circularly polarized electroluminescence from liquid-crystalline chiral polyfluorenes. Adv. Mater. 12, 362-365 (2000)

48 Meskers, S. C. J., Peeters, E., Langeveld-Voss, B. M. W. \& Janssen, R. A. J. Circular polarization of the fluorescence from films of poly(p-phenylene vinylene) and polythiophene with chiral side chains. Adv. Mater. 12, 589-594 (2000).

49 Wilson, J. N., Steffen, W., McKenzie, T. G., Lieser, G., Oda, M., Neher, D. \& Bunz, U. H. F. Chiroptical properties of poly( $p$-phenyleneethynylene) copolymers in thin films large g-values. J. Am. Chem. Soc. 124, 6830-6831 (2002).

50 Meskers, S. C. J., Dekkers, H. P. J. M., Rapenne, G. \& Sauvage, J. -P. Chiroptical properties of an optically pure dicopper(I) trefoil knot and its enantioselectivity in luminescence quenching reactions. Chem.-Eur. J. 6, 2129-2134 (2000)

51 Schaffner-Hamann, C., von Zelewsky, A., Barbieri, A., Barigelletti, F., Muller, G. Riehl, J. P. \& Neels, A. Diastereoselective formation of chiral tris-cyclometalated iridium (III) complexes: characterization and photophysical properties. J. Am. Chem. Soc. 126, 9339-9348 (2004).

52 Oyler, K. D., Coughlin, F. J. \& Bernhad, S. Controlling the helicity of 2,2'-bipyridy ruthenium(II) and zinc(II) hemicage complexes. J. Am. Chem. Soc. 129, 210-217 (2007)

53 Harada, T., Nakano, Y., Fujiki, M., Naito, M., Kawai, T. \& Hasegawa, Y. Circularly polarized luminescence of $\mathrm{Eu}(\mathrm{III})$ complexes with point- and axis-chiral ligands dependent on coordination structures. Inorg. Chem. 48, 11242-11250 (2009).

54 Kaseyama, T., Furumi, S., Zhang, X., Tanaka, K. \& Takeuchi, M. Hierarchical assembly of a phthalhydrazide-functionalized helicene. Angew. Chem. Int. Ed. Engl. 50, 3684-3687 (2011).

55 Sawada, Y., Furumi, S., Takai, A., Takeuchi, M., Noguchi, K. \& Tanaka, K. Rhodium catalyzed enantioselective synthesis, crystal structures, and photophysical properties of helically chiral 1,1'-bitriphenylenes. J. Am. Chem. Soc. 134, 4080-4083 (2012).

56 Chen, S. H., Katsis, D., Schmid, A. W., Mastrangelo, J. C., Tsutsui, T. \& Blanton, T. N. Circularly polarized light generated by photoexcitation of luminophores in glassy liquid-crystal films. Nature 397, 506-508 (1999).

57 Voigt, M., Chambers, M. \& Grell, M. On the circular polarization of fluorescence from dyes dissolved in chiral nematic liquid crystals. Chem. Phys. Lett. 347, 173-177 (2001).

58 Bobrovsky, A. Y., Boiko, N. I., Shibaev, V. P. \& Wendorff, J. H. Cholesteric mixtures with photochemically tunable, circularly polarized fluorescence. Adv. Mater. 15, 282-287 (2003).

59 Woon, K. L., O'Neill, M., Richards, G. J., Aldred, M. P., Kelly, S. M. \& Fox, A. M. Highly circularly polarized photoluminescence over a broad spectral range from a calamitic, hole-transporting, chiral nematic glass and from an indirectly excited dye. Adv. Mater. 15, 1555-1558 (2003)

60 Riehl, J. P. \& Richardson, F. S. Circularly polarized luminescence spectroscopy. Chem. Rev. 86, 1-16 (1986).

61 Furumi, S. \& Sakka, Y. Circularly polarized laser emission induced by supramolecular chirality in cholesteric liquid crystals. J. Nanosci. Nanotechnol. 6, 1819-1822 (2006).

62 Furumi, S. \& Sakka, Y. Chiroptical properties induced in chiral photonic-bandgap liquid crystals leading to a highly efficient laser-feedback effect. Adv. Mater. 18, 775-780 (2006).

63 Kato, T. Self-assembly of phase-segregated liquid crystal structures. Science 295 2414-2418 (2002).

64 Kato, T., Mizoshita, N. \& Kishimoto, K. Functional liquid-crystalline assemblies: selforganized soft materials. Angew. Chem. Int. Ed. 45, 38-68 (2006).

65 Furumi, S., Yokoyama, S., Otomo, A. \& Mashiko, S. Control of photonic bandgaps in chiral liquid crystals for distributed feedback effect. Thin Solid Films 499, 322-328 (2003)

66 Wysocki, J. J., Adams, J. \& Haas, W. Electric-field-induced phase change in cholesteric liquid crystals. Phys. Rev. Lett. 20, 1024-1025 (1968).

67 Kahn, F. J. Electric-field-induced color changes and pitch dilation in cholesteric liquid crystals. Phys. Rev. Lett. 24, 209-212 (1970).

68 Furumi, S., Yokoyama, S., Otomo, A. \& Mashiko, S. Phototunable photonic bandgap in a chiral liquid crystal laser device. Appl. Phys. Lett. 84, 2491-2493 (2004)

69 Haas, W., Adams, J. \& Wysocki, J. Interaction between UV radiation and cholesteric liquid crystals. Mol. Cryst. Liq. Cryst. 7, 371-379 (1969).

70 Lin, T. -H., Chen, Y. -J., Wu, C. -H., Fuh, A. Y. -G., Liu, J. -H. \& Yang, P. -C. Cholesteric liquid crystal laser with wide tuning capability. App. Phys. Lett. 86, 161120 (2005).

71 Kurihara, S., Hatae, Y., Yoshioka, T., Moritsugu, M., Ogata, T. \& Nonaka, T. Phototuning of lasing from a dye-doped cholesteric liquid crystals by photoisomerization of a sugar derivative having plural azobenzene groups. Appl. Phys. Lett. 88, 103121 (2006).

72 Chilaya, G., Chanishvili, A., Petriashvili, G., Barberi, R., Bartolino, R., Cipparrone, G., Mazzulla, A. \& Shibaev, P. V. Reversible tuning of lasing in cholesteric liquid crystals controlled by light-emitting diodes. Adv. Mater. 19, 565-568 (2007).

73 Shank, C. V., Bjorkholm, J. E. \& Kogelnik, H. Tunable distributed-feedback dye laser. Appl. Phys. Lett. 18, 395-396 (1971).

74 Huang, Y., Zhou, Y. \& Wu, S. T. Spatially tunable laser emission in dye-doped photonic liquid crystals. Appl. Phys. Lett. 88, 011107 (2006).

75 Chanishvili, A., Chilaya, G., Petriashvili, G., Barberi, R., Bartolino, R., Cipparrone, G., Mazzulla, A. \& Oriol, L. Lasing in dye-doped cholesteric liquid crystals: two new tuning strategies. Adv. Mater. 16, 791-795 (2004).

76 Sonoyama, K., Takanishi, Y., Ishikawa, K. \& Takezoe, H. Position-sensitive cholesteric liquid crystal dye laser covering a full visible range. Jpn. J. Appl. Phys. 46, L874-L876 (2007).

77 Huang, Y., Chen, L. P., Doyle, C., Zhou, Y. \& Wu, S. T. Spatially tunable laser emission in dye-doped cholesteric polymer films. Appl. Phys. Lett. 89, 111106 (2006).

78 Manabe, T., Sonoyama, K., Takanishi, Y., Ishikawa, K. \& Takezoe, H. Toward practica application of cholesteric liquid crystals to tunable lasers. J. Mater. Chem. 18, 3040-3043 (2008)

79 Furumi, S., Yokoyama, S., Otomo, A. \& Mashiko, S. Study on laser action from UV-curable chiral nematic liquid crystals. Thin Solid Films 438, 423-427 (2003).

80 Furumi, S. \& Tamaoki, N. Glass-forming cholesteric liquid crystal oligomers for new tunable solid-state laser. Adv. Mater. 22, 886-891 (2010).

81 Xia, Y., Gates, B., Yin, Y. \& Lu, Y. Monodispersed colloidal spheres: old materials with new applications. Adv. Mater. 12, 693-713 (2000).

82 Colvin, V. L. From opals to optics: colloidal photonic crystals. MRS Bull. 26, 637-641 (2001).

83 Ozin, G. A. \& Yang, S. M. The race for the photonic chip: colloidal crystal assembly in silicon wafers. Adv. Funct. Mater. 11, 95-104 (2001).

84 Yan, Q., Wang, L. \& Zhao, X. S. Artificial defect engineering in three-dimensional colloidal photonic crystals. Adv. Funct. Mater. 17, 3695-3706 (2007).

85 Sato, O., Kubo, S. \& Gu, Z. Z. Structural color films with lotus effects, Superhydrophilicity, and tunable stop-bands. Acc. Chem. Res. 4, 1-10 (2009).

86 Harun-Ur-Rashid, M., Seki, T. \& Takeoka, Y. Structural colored gels for tunable soft photonic crystals. Chem. Rec. 9, 87-105 (2009)

87 Furumi, S, Fudouzi, H. \& Sawada, T. Self-organized colloidal crystals for photonics and laser applications. Laser \& Photon. Rev. 4, 205-220 (2010).

88 Aguirre, C. I., Reguera, E. \& Stein, A. Tunable colors in opals and inverse opa photonic crystals. Adv. Funct. Mater. 20, 2565-2578 (2010).

89 Galisteo-López, J. F., Ibisate, M., Sapienza, R., Froufe-Pérez, L. S., Blanco, Á. \& López, C. Self-assembled photonic structures. Adv. Mater. 23, 30-69 (2011).

90 Kim, S.-H., Lee, S. Y., Yang, S. M. \& Yi, G. R. Self-assembled colloidal structures for photonics. NPG Asia Mater. 3, 25-33 (2011).

91 Ge, J. \& Yin, Y. Responsive photonic crystals. Angew. Chem. Int. Ed. 50, 1492-1522 (2011)

92 Mayoral, R., Requena, J., Moya, J. S., López, C., Cintas, A., Miguez, H., Meseguer, F. Vázquez, L., Holgado, M. \& Blanco, A. 3D long-range ordering in ein $\mathrm{SiO}_{2}$ submicrometer-sphere sintered superstructure. Adv. Mater. 9, 257-260 (1997).

93 Furumi, S., Fudouzi, H., Miyazaki, H. T. \& Sakka, Y. Flexible polymer colloidalcrystal lasers with a light-emitting planar defect. Adv Mater 19, 2067-2072 (2007).

94 Furumi, S., Kanai, T. \& Sawada, T. Widely tunable lasing in a colloidal crystal gel film permanently stabilized by an ionic liquid. Adv. Mater. 23, 3815-3820 (2011). 
95 Furumi, S. Recent advances in polymer colloidal crystal lasers. Nanoscale 4, 5564-5571 (2012)

96 Fudouzi, H. \& Xia, Y. Colloidal crystals with tunable colors and their use as photonic papers. Langmuir 19, 9653-9660 (2003).

97 Shkunov, M. N., Vardeny, Z. V., DeLong, M. C., Polson, R. C., Zakhidov, A. A. \& Baughman, R. H. Tunable, gap-state lasing in switchable directions for opal photonic crystals. Adv. Funct. Mater. 12, 21-26 (2002).

98 Lawrence, J. R., Ying, Y., Jiang, P. \& Foulger, S. H. Dynamic tuning of organic lasers with colloidal crystals. Adv. Mater. 18, 300-303 (2006).

99 Jin, F., Li, C. F., Dong, X. Z., Chen, W. Q. \& Duan, X. M. Laser emission from dyedoped polymer film in opal photonic crystal cavity. Appl. Phys. Lett. 89, 241101 (2006).

100 Yamada, H., Nakamura, T., Yamada, Y. \& Yano, K. Colloidal-crystal laser using monodispersed mesoporous silica spheres. Adv. Mater. 21, 4134-4138 (2009).

$101 \mathrm{Kim}, \mathrm{S}$. H., Kim, S. H., Jeong, W. C. \& Yang, S. M. Low-threshold lasing in 3D dyedoped photonic crystals derived from colloidal self-assemblies. Chem. Mater. 21, 4993-4999 (2009).

102 Ozaki, R., Matsui, T., Ozaki, M. \& Yoshino, K. Electrically color-tunable defect mode lasing in one-dimensional photonic-band-gap system containing liquid crystal. Appl. Phys. Lett. 82, 3593-3595 (2003).

103 Yoon, J., Lee, W., Caruge, J. M., Bawendi, M., Thomas, E. L., Kooi, S. \& Prasad, P. N. Defect-mode mirrorless lasing in dye-doped organic/inorganic hybrid one-dimensional photonic crystal. Appl. Phys. Lett. 88, 091102 (2006).

104 Shung, K. W. K. \& Tsai, Y. C. Surface effects and band measurements in photonic crystals. Phys. Rev. B. 48, 11265 (1993).
105 Furumi, S., Fudouzi, H. \& Sawada, T. Dynamic photoswitching of micropatterned lasing in colloidal crystals by the photochromic reaction. J. Mater. Chem. 22, 21519 (2012).

106 Sawada, T., Suzuki, Y., Toyotama, A. \& lyi, N. Quick fabrication of gigantic singlecrystalline colloidal crystals for photonic crystal applications. Jpn. J. Appl. Phys. 40, L1226-L1228 (2001).

107 Toyotama, A., Kanai, T., Sawada, T., Yamanaka, J., Ito, K. \& Kitamura, K. Gelation of colloidal crystals without degradation in their transmission quality and chemical tuning. Langmuir 21, 10268-10270 (2005).

108 Kanai, T., Sawada, T., Toyotama, A. \& Kitamura, K. Air-pulse-drive fabrication of photonic crystal films of colloids with high spectral quality. Adv. Funct. Mater. 15, 25-29 (2005)

109 Kanai, T., Yamamoto, S. \& Sawada, T. Swelling of gel-immobilized colloidal photonic crystals in ionic liquids. Macromolecules 44, 5865-5867 (2011).

110 Dowling, J. P., Scalora, M., Bloemer, M. J. \& Bowden, C. M. The photonic band-edge laser -a new approach to gain enhancement. J. Appl. Phys. 75, 1896-1899 (1994).

111 Sakoda, K. Enhanced light amplification due to group-velocity anomaly peculiar to two- and three-dimensional photonic crystals. Opt. Express 4, 167-176 (1999).

112 Finkelmann, H., Kim, S. T., Muñoz, A., Palffy-Muhoray, P. \& Taheri, B. Tunable mirrorless lasing in cholesteric liquid crystalline elastomers. Adv. Mater. 13, 1069-1072 (2001).

113 Weinberger, M. R., Langer, G., Pogantsch, A., Hasse, A., Zojer, E. \& Kern, W. Continuously color-tunable rubber laser. Adv. Mater. 16, 130-133 (2004).

114 Görrn, P., Lehnhardt, M., Kowalsky, W., Riedl, T. \& Wagner, S. Elastically tunable self-organized organic lasers. Adv. Mater. 23, 869-872 (2011).

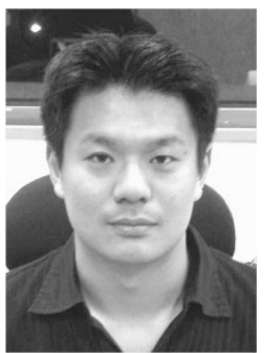

Seiichi Furumi is a principal researcher of the National Institute for Materials Science (NIMS) in Japan. He received PhD degree from the Tokyo Institute of Technology in 2001 under the supervision of Prof. Kunihiro Ichimura. From 1998 to 2001, he was also at the Japan Society for the Promotion of Science (JSPS) Research Fellow for Young Scientist (DC1). After his PhD studies, he joined in the Communications Research Laboratory as a Postdoctoral Researcher. In 2004, he held a permanent position at NIMS. From 2010, he concurrently started a research project as a PRESTO Researcher of the Japan Science and Technology Agency (JST). Since 2012, he has also served as an Associated Professor of Department of Materials Science and Engineering in the University of Tsukuba. He received the Hitachi Chemical Award from the Society of Polymer Science, Japan (SPSJ) in 2011. Currently, his research interest is the development of polymer materials for photonics applications. 